\section{Abstracts of the 1st Congress of the International Academy of Digital Pathology}

\section{August 3-5, 2011 Quebec city, Canada}

The challenges of implementing a "patientoriented" telepathology network; the Eastern Quebec telepathology project experience

\section{Bernard Têtu}

RUIS-Laval University Telepathology Project, Québec, Canada

Background: The Eastern Québec telepathology project was initiated by the Laval University Integrated University Health Network (in French: RUIS: Réseau Universitaire Intégré en Santé). The RUIS was created by the Québec ministry of health as an authority of coordination and consensus which mandate is to encourage the integration of health cares, teaching and research activities in the institutions under the umbrella of the faculty of medicine. This project is aimed at providing uniform diagnostic telepathology services in a territory of $408,760 \mathrm{~km}^{2}$ with a population of 1.7 millions inhabitants and which density, in certain areas, is as low as 0.4 inhabitant $/ \mathrm{km}^{2}$.

Clinical context: Many surgeons in smaller community hospitals must postpone surgeries requiring a frozen-section or to transfer certain patients to regional hospitals because of the lack of stable pathology coverage. Younger pathologists in early practice feel insecure and are often reluctant to work alone because of the impossibility to rapidly obtain a second opinion from a colleague and can hardly be absent without disturbing the surgical unit organization. In this context, the ministry of heath and Canada Health Infoway decided to financially support this innovative initiative with the objective of providing the population with uniform pathology services on the territory. The project is particularly timely because of the reports of two recent commissions of inquiry in Canada recommending the deployment of telepathology to help providing professional support and improve the quality of cares in Canada.
Scope of the project: this is a "patient-oriented" project aimed at providing surgeons and pathologists with frozen sections and second opinions anywhere and anytime on the whole territory of the RUIS-Laval University. Each hospital is equipped with a whole slide scanner, a gross station, a videoconferencing device and with a viewer and an image sharing solution. The project has been implemented in 21 sites, of which 6 are devoid of pathology laboratory. Of the remaining 15 sites, 6 have one pathologist, 7 have 2 or more pathologists and two have no pathologist. Change management is the major challenge encountered in the implementation of this network. Basically, we found that 1) Although the surgeons of the 6 hospitals devoid of pathology laboratory and the 2 devoid of pathologists are motivated by the potential of the technology, they must get used to request services that were unavailable for so many years; 2) The network must adapt to a changing situation occurring in the course of the deployment, such as the moving and retirement of pathologists; 3) This unique network combining centers with or without laboratory requires the development of innovative solutions (new report format, gross station for large specimens and discussions with the surgeon); 4) Pathologists used to send consultations by mail must get used to send and read images; 5) The network must be developed within each region because University hospital are unable to absorb an increased workload; 6) Surgeons and pathology personnel must develop new working methods; 7) Technicians in hospitals without pathology laboratory have limited experience with histology techniques.

Conclusion: The Eastern Québec telepathology network has been designed to improve medical care to patients in this territory. However, the project team is confronted with major challenges mostly related to change management and requiring innovative solutions.

\section{Non-destructive digital pathology using Light-CT'M on fresh and fixed tissue: Initial experience for clinical and research applications}

\section{Michael Riben}

The University of Texas MD Anderson Cancer Center, Houston, TX, USA 
Background: It is increasingly important to maximize the volume and quality of tissue obtained for both clinical and research use, particularly in the current push for delivering personalized cancer therapy. Given the expanding potential uses for collected tissue, minimizing consumption while confirming the histologic diagnosis and assessing the quality of tissue obtained, both of which plague current methods such as frozen section or cytologic assessment, is critical. To this end, we have begun testing a novel commercial device for full-field optical coherence tomography called Light-CT ${ }^{\mathrm{TM}}$ which facilitates rapid non-destructive histologic digital imaging of both fresh and fixed tissue. The technique is based on the principle of white light interferometry and generates quick image slices in three-dimensions of the specimen. Our initial experience includes evaluation of the ability to use the images for quality control and the potential for diagnostic assessment.

Methods: We obtained residual tissue from gastrointestinal tract, breast, and skin for imaging. Full field and virtual sectioned slides of tissues at a variety of depths were acquired using a $10 \times / 0.3$ numerical aperture immersion objective. Images were processed and visualized in real-time. The imaged tissue was submitted for traditional histology, after which comparison of the Light-CT ${ }^{\mathrm{TM}}$ image and the glass slide were undertaken.

Results: Initial experience show architectural and morphologic similarity between the Light-CT image and histologic preparations. Distinct morphologic criteria includes pixel intensity, patterns which correlate with histologic features, and architectural variability. Together, these provide adequate information for a quality determination. Image correlation with the stained H\&E section highlights image features with associated histologic features including architecture, tumor invasion, tissue reaction, and normal structures.

Conclusions: Light-CT ${ }^{\mathrm{TM}}$ represents a paradigm shift for histologic assessment of both fresh and fixed tissue. We have shown that the images produced show great potential for use-cases in which pattern recognition and architectural assessment are used to evaluate both for quality of tissue obtained and diagnostic criteria. Future work includes assessing molecular markers after imaging.

\section{Standardization efforts of digital pathology in Europe}

\section{Marcial García Rojo}

\section{Hospital General Unviersitario de Ciudad Real, Ciudad Real, Spain}

EURO-TELEPATH stands for "Telepathology Network in Europe", a European COST Action (http:// www.conganat.org/eurotelepath/) that started in 2007 and will end in November 2011. This project is aimed to foster collaboration between European research groups working in an adequate technological framework for the management of multimedia electronic healthcare records, in informatics applied to Anatomic Pathology, and, most importantly in IT standards applied to digital medical images, collaborate with international standardization bodies.

Working Group 1 "Business Modeling in Pathology" performed a comparison study between different notations in business modeling (Business Process Modeling Notation - BPMN, Event Process Chain EPC, and UML Activity Diagram). They have concluded that BPMN is the modeling notation which is clearer and more understandable to pathologists. EPC is harder for pathologists to read and understand. It is probably because EPC is not as popular as BPMN and UML AD, so pathologists are not so familiar with the concept of event-driven process.

Main pathology processes - Frozen Study, Formalin Fixed Specimen Study, Telepathology, Cytology, Autopsy - are now complete. Further work will be needed to modify the processes according to feedbacks we have gotten from the pathologists. These results can be beneficial to improve the organization of digitizing centers, since they can used to create models and protocols enabling telepathology international network websites for teleconsultation services. In the next future, this work can also be applied in the documentation of pathology processes described in many standardization documents (DICOM, HL7, IHE).

Working Group 2 has been dedicated to Informatics Standards in Pathology, and promoting their application in Pathology Departments in European institutions. The main research is focused in: A) Advances in Integrating Healthcare Enterprise (IHE)- Anatomic Pathology: Anatomic Pathology Workflow (APW) within the hospital, Anatomic Pathology Reporting to Public Health (ARPH) and Anatomic Pathology Structured Report (APSR), based in HL7 Clinical Docu- 
ment Architecture (CDA). B) The practical application of DICOM standard (medical image) in Pathology. C) Image compression. D) Semantic interoperability: PathLex and SNOMED CT in Pathology.

Image compression research discussion has been performed in collaboration with JPEG committee. As a conclusion of this discussion, it seems that JPEG 2000 image compression has proved to be a most efficient image compression standard, but is has not become widely implemented. JPEG commitee is working in the development of a new compression method, JPEG XR, mainly aimed at those digital photography applications where JPEG 2000 penetration has been limited due to complexity reasons, and they are also interested in evaluating Advanced Image Coding (AIC) in medical images.

\section{Toward standardization: Color and image quality validation in Whole Slide Imaging (WSI)}

Yukako Yagi, Noriaki Hashimoto and Pinky

Bautista

Department of Pathology, Harvard Medical School, Massachusetts General Hospital, Boston, MA, USA

Background: Standardization of the image quality and the color displayed by digital slides are important aspects of digital pathology implementation. While the most common reason for the variations of color and image quality is the variance in the protocols and practices in the histology lab, the image displayed can also be affected by variation in capture parameters (for example, focus, illumination and filters), image processing and display factors in the digital systems themselves. It is difficult to identify which exactly cause the problem. We have developed the methodology to standardize color and evaluate image quality of any WSI.

Design: Two types of calibration slides were developed in our laboratory: color and image quality calibration slides. These slides were scanned using different scanners. The color information from the scanned image of the color calibration slide was used to characterize the color settings of the scanners. The effectiveness of the proposed color standardization and image quality evaluation algorithms was demonstrated using the image quality evaluation slide, which is a ideally stained embryo tissue slide.
Results: The scanned images showed varying color appearance on the same monitor. However, after the implementation of the color standardization algorithm the color variance was minimized. Moreover, there was a good correlation between the results of the proposed image quality evaluation method with the evaluation results by human eyes. We have developed a tentative protocol for color standardization and image quality validation.

Conclusions: Initial results of the standardization experiments are promising although there are still several factors that we need to consider. We have been conducting a series of research towards Standardization. However, to promote the importance to have standardized color of image and high quality of image and also standardize validation protocol, the collaboration between Academic, Industry, Governmental organization may be necessary.

\section{DICOM working group activity}

\section{Michael Meissner}

\section{Co-Chair of DICOM WG26, USA}

DICOM WG26 has successfully completed two supplements, Supplement 122 targeting primarily LIS vendors to exchange specimen information and supplement 145 primarily targeting imaging vendors to exchange WSI information. Without any doubt, those two are major milestones in the process of establishing a framework for standardized digital Pathology Informatics. With standards being established, it is now up to the vendors to implement those but even more so up to the pathology institutions to demand vendors to support of these standards so that the adoption can occur more quickly. Having those standards is important but even if those were supported by most vendors todays, standardized Pathology Informatics needs more: i.e., most devices today do not support device independent image information and support ICC profiles is critically important for the development of image algorithms that can be applied across different vendors' images. Without it, development will be slow and availability of such algorithms will not be as widespread as it could potentially be. DICOM WG26 continues its efforts and activities to establish and enhance interoperability, most recently targeting the adoption of DICOM Worklists to define procedures and procedure steps that allow devices in 
the pathology space to communicate respective status. In this session, we will provide an update on the ongoing activities in DICOM WG26 as well as seek ideas for additional topics that WG26 could/should tackle in the future. While Informatics is not something pathologists are very familiar with/commonly exposed to, the future of pathology lies heavily in Pathology Informatics and DICOM WG26 would benefit from more active end user (pathologists) participation which we would like to improve.

This talk will give an overview of the accomplishments of DICOM WG26, current activities, and potential future efforts. We believe educating about such progress and getting more active participation by various parties is a key ingredient to moving Pathology into the Digital Age.

\section{Validating Whole Slide Imaging for diagnostic purposes in pathology: Recommendations of the College of American Pathologists (CAP) pathology and laboratory quality center}

Andrew J. Evans ${ }^{1}$, John H. Sinard ${ }^{2}$, Lisa A. Fatheree ${ }^{3}$, Walter H. Henricks ${ }^{4}$, Alexis B. Carter ${ }^{5}$, Lydia Contis ${ }^{6}$, Bruce A. Beckwith ${ }^{7}$, Christopher N. Otis ${ }^{8}$, James MacDonald ${ }^{3}$, Anil V. Parwani ${ }^{6}$ and Liron Pantanowitz ${ }^{6}$

${ }^{1}$ Laboratory Medicine Program, University Health Network, Toronto, ON, Canada

${ }^{2}$ Department of Pathology, Yale University School of Medicine, New Haven, CT, USA

${ }^{3}$ College of American Pathologists, Northfield, IL, USA

${ }^{4}$ Pathology and Laboratory Medicine Institute, Cleveland Clinic, Cleveland, OH, USA

${ }^{5}$ Department of Pathology and Laboratory Medicine, Emory University, Atlanta, GA, USA

${ }^{6}$ North Shore Medical Center Salem Hospital, Salem, MA, USA

${ }^{7}$ Department of Pathology, University of Pittsburgh Medical Center, Pittsburgh, PA, USA

${ }^{8}$ Department of Pathology, Baystate Medical Center, Tufts University School of Medicine, Springfield, MA, USA

Introduction: There is an increasing interest in using whole slide imaging (WSI) for diagnostic purposes (primary and/or consultation). An important question is whether WSI can replace conventional light microscopy as the method by which pathologists review histologic sections and/or cytology slides to render diagnoses. Validation of WSI is crucial to ensure that diagnostic performance based on digitized slides is at least equivalent to that of glass slides and light microscopy. Currently, there are no standardized guidelines regarding validation of WSI for diagnostic use.

Methods: The CAP Pathology and Laboratory Quality Center convened a non-vendor panel from North America with expertise in digital pathology. A total of 611 international publications that met search term requirements were identified. Papers outside the scope and related solely to technical components, education and image analysis were subsequently excluded. Publication year, author country, WSI clinical application, number of observers and cases used, validation method and outcome measurement were recorded. Twenty two articles cleared the work group requirements and were submitted for quality of evidence grading by a contracted methodologist. Draft recommendations were developed from this comprehensive literature review and panel consensus. Final recommendations will be completed after a public comment period.

Results and conclusions: Validation of WSI is essential to ensure that pathologists using this technology for diagnostic purposes will consistently make the same clinical interpretation as they would from glass slides and a light microscope. Validation should address both technical and interpretative components to demonstrate that the WSI system under review produces acceptable digital slides for diagnostic interpretation. A pathologist must be involved in the validation process. In order to simulate actual clinical practice, a validation sample set should be of a minimum size and include the spectrum of specimen types and diagnoses likely to be encountered with the intended clinical use. Measurable outcomes should document diagnostic concordance (accuracy) between digital and glass slides for the same observer (intra-observer variability) with a reasonable washout period. Approved clinical use of WSI should be limited to the conditions under which validation occurred. 


\section{Z-axis challenges in Whole Slide Imaging (WSI) telepathology}

Ronald S. Weinstein, Anna R. Graham, Fangru Lian and Achyut K. Bhattacharyya

Department of Pathology, University of Arizona College of Medicine and Arizona Telemedicine Program, Tucson, AZ, USA

Surgical pathology diagnostic accuracy studies comparing telepathology (i.e., digital imaging, whole slide imaging, virtual microscopy) and conventional light microscopy often show slightly lower diagnostic accuracy for telepathology. When the differences in diagnostic accuracy are not statistically significant, isolated diagnostic errors can still have serious implications for individual patients.

We surveyed experienced telepathologists in order to identify potential sources of diagnostic difficulty, especially for cases in which specimens are imaged in a single focal plane. The participating pathologists had experience with both digital robotic telepathology and whole slide imaging telepathology. The pathologists listed potential "problem" surgical pathology cases where through-focal imaging (imaging of multiple planes in the Z-axis) could be especially important.

In this survey, the following types of cases were identified as potentially dependent on imaging multiple focal planes (Z-axis imaging). In surgical pathology, identifying and classifying microorganisms by conventional light microscopy can be challenging. This is especially so for certain bacteria such as Helicobacter pylori, because of their small size and shape, fungi, and parasites. The identification of viral inclusions in single focal plane digital images is challenging. Interpretation of renal biopsies for transplantation rejection benefits from examining the distribution of lymphocytes in multiple planes. Also, in surgical pathology, identifying crystals in single plane digital images of biopsies can be difficult. In cancer cases, identification of mitotic figures in surgical pathology sections, and differentiating normal from abnormal mitotic figures, can be problematic. There are many examples of cytopathology specimens benefiting from multiple focal plane imaging. Examples of cytology cases that might benefit from digital imaging in multiple planes include PAP smears, and body fluids in which there might be a tendency for cells to aggregate into balls. Cells in FNAs (fine needle aspirates) can also "ball up" into large aggregates greater in diameter than the thickness of a single Z-axis focal plane digital image. Identification of crystals and casts in urine specimens can be problematic with single Z-axis plane viewing. Hematopathology studies, using smears, can be challenging, because of the need for imaging in exquisite detail, often requiring oil-immersion imaging with a through-focal series of images.

Interestingly, these "consensus" diagnostic accuracy challenges often correspond to problem areas that are underrepresented in the digital pathology literature. This may reflect the well-known predilection of clinical investigators to study questions that are likely to yield a positive outcome. Before digital pathology is incorporated into routine practice, guidelines for handling of potential "problem cases" should be incorporated into standard operating procedures.

\section{Proof of principle: Colocalization of pan cytokeratins (AE1/AE3), pan cytokeratin (PCK26), and cytokeratin 8/18 using an Integrated Sequential Staining and Imaging Device}

Judit Zubovits ${ }^{1}$, Kashan Shaikh ${ }^{3}$, Alex Corwin ${ }^{3}$, Dan Wang ${ }^{4}$, Sean Dinn², Gina Clarke ${ }^{4}$, Chris Peressotti ${ }^{4}$, Zhengyu Pang ${ }^{2}$, Robert Filkins ${ }^{2}$ and Martin J. Yaffe ${ }^{4}$ ${ }^{1}$ Department of Anatomic Pathology, Sunnybrook
Health Sciences Centre, Toronto, Canada
${ }^{2}$ Diagnostic and Biomedical Technologies, GE
Global Research Center, Niskayuna, NY, USA
${ }^{3}$ Electrical Technologies and Systems, GE Global
Research Center, Niskayuna, NY, USA
${ }^{4}$ Ontario Institute for Cancer Research and Sunny-
brook Research Institute, Toronto, Ontario, Canada

Background: Co-localization of biochemical and molecular markers at the cellular and subcellular levels remains an active area of research and development within image analysis. Cytokeratins (CK) are a family of about 20 different water-soluble proteins that form the cytoskeleton of epithelial cells. All of them are used in the clinical lab to recognize epithelial cells. We demonstrate a unique optofluidic instrument and a multiplexing immunohistochemistry technique for sequentially labeling different proteins on the same section of breast cancer and illustrate its use in analyzing the co-localization between 3 fluorescent cytokeratin markers. 
Methods: Antibodies: Pan cytokeratin (panCK) AE1/AE3 (Catalog\# M3515, DAKO, Carpinteria, CA); cytokeratin 8/18 (CK8/18) (Clone K8.8+DC 10), (Catalog\# MS-1603-P0, Lab Vision Fremont, CA); panCK (clone PCK26) (Catalog\# C1801, Sigma, St. Louis, MS). A de-identified breast cancer sample was collected from the archives of Anatomic Pathology Department at Sunnybrook Health Sciences Centre. Biomarker multiplexing was performed using an automatic, sequential staining and imaging system. Images were registered, and the autofluorescence signal removed. Co-localization was quantified on a pixel by pixel basis using Pearson's correlation coefficient (r). A mean value of $r$ was obtained over multiple regions of interest (ROIs) on the section.

Results: Initially, to estimate operational stability, perfectly correlated samples were obtained by imaging the same section but using different exposure times. Due to noise in the image acquisition system, the $\mathrm{r}$ value was not 1 but $0.988 \pm 0.001$. A total of 14 ROIs each measuring 758 microns by 758 microns were acquired on a single section of invasive ductal carcinoma of the breast. The correlations between CK8/18 and panCK(PCK26), panCK(AE1/AE3) and panCK(PCK26), panCK(AE1/AE3) and CK8/18 were $0.959 \pm 0.016,0.918 \pm 0.03,0.904 \pm 0.029$, respectively. Since CK8/18 recognizes all simple epithelia including glandular epithelium, it is very likely that $\mathrm{CK} 8 / 18$ is the primary $\mathrm{CK}$ in this breast cancer specimen, or all other types of CKs are highly correlated to the expression of $\mathrm{CK} 8 / 18$.

Conclusion: Our work demonstrates a novel and versatile system for co-localization studies that can be easily adapted to work with existing commercially available antibodies in a translational research setting.

\section{Benefits of a two resolution strategy for analyzing virtual slides of immunostained tumors}

Catherine Bor ${ }^{1,2}$, Paulette Herlin ${ }^{1}$, Nicolas Elie ${ }^{1}$ and Benoît Plancoulaine ${ }^{1}$

${ }^{1}$ GRECAN, EA 1772, IFR146 ICORE, Caen University and François Baclesse Cancer Centre, Caen, France

${ }^{2}$ Pathology Department, François Baclesse Cancer

Centre, Caen, France
Background: The quantitative estimation of immunostained biomarkers on histological sections of tumors provides oncologists with prognostic and therapeutic indicators. To take into account the frequent structural and functional heterogeneity of tumors, the study of sampled representative whole sections of the tumors is mandatory. The fully automated analysis of high resolution virtual slides offers a new opportunity in this context. Nevertheless, due to their huge size, it is necessary to define a swift and efficient strategy of analysis.

Methods: The proposed approach includes the carefull subsampling of the high resolution image ( 0.5 or $0.25 \mu \mathrm{m}$ ) up to the critical resolution of $4 \mu \mathrm{m}$ and the combined automatic analysis at both levels. The collection of data dealing with the whole tumor tissue is done at the low resolution level and on the whole image (mean and maximum density of the marker under study and characterization of its distribution). The additional information concerning the cellular and subcellular parameters is done at high resolution, taking advantage of a cloning of the structures of interest or of a systematic sampling of tissue details. The low resolution level allows to isolate the tissue compartments and immunostained markers of interest, while the high resolution level helps in refining the segmentation done at low resolution. The superimposition, on the full resolution image, of the upscaled segmentation masks obtained at low resolution, allows pathologist' visual control. The intersection of these masks with the results of an interactive reference marking of a stereological grid, done at full resolution, leads to an evaluation of the quality of the automatic analysis method which was implemented.

Results: The two resolution analysis which is illustrated through several examples (quantification of nuclear markers and blood vessels) runs on commercially available personal computers and allows collection of data from the whole tumor sections in a very short delay, compatible with the routine practice.

Conclusions: Thanks to this simple and easy to implement strategy, the swift analysis of various pathological virtual slides seems at hand. 


\section{Mitotic figure recognition: Agreement among pathologists and computerized detector}

Christopher Malon ${ }^{2}$, Elena Brachtel ${ }^{5}$, Eric Cosatto ${ }^{2}$, Hans Peter $\mathrm{Graf}^{2}$, Atsushi Kurata ${ }^{3}$, Masahiko Kuroda $^{3}$, John S. Meyer ${ }^{4}$, Akira Saito ${ }^{1}$, Shulin $\mathrm{Wu}^{5}$, and Yukako Yagis

${ }^{1}$ Innovative Service Solutions Division, NEC Corporation, USA

${ }^{2}$ Department of Machine Learning, NEC

Laboratories, USA

${ }^{3}$ Department of Pathology, St. Luke's Hospital

${ }^{4}$ Department of Molecular Pathology, Tokyo Medical University, Tokyo, Japan

${ }^{5}$ Department of Pathology, Massachusetts General Hospital and Harvard Medical School, Boston, MA, USA

Background: We compare three pathologists' assessments of mitotic figures and develop a system to recognize mitosis using machine learning. Identification of mitotic figures is a laborious task that is essential to grading and prognosis for breast cancer. A mitotic count provides one of the three components of the Bloom- Richardson grade for invasive carcinoma [2]. Yet, according to several studies ([1,3 and 4], and [1]), pathologist agreement for mitotic grade is rather modest, with [3] reporting agreement of $\kappa=0.38$. An automated mitosis count could accelerate a pathologist's work while improving reproducibility.

Methods: We ask three pathologists to classify 4,204 sites as "mitosis", "might or might not be mitosis," or "not mitosis". We compute statistics of agreement among these pathologists. Based on majority-agreed sites, we train a computer to recognize the mitotic figures. The computer uses image analysis heuristics to extract candidates, then combines manually designed features with features automatically learned by a convolutional neural network, to make a final decision for each candidate point, using a support vector machine.

Results: The machine's performance falls within the range of the humans' performance if "maybe" labels must be committed to be affrmative or negative:

Among the three pathologists, we observe pairwise agreements of $\kappa \mathrm{AB}=0.13, \kappa \mathrm{AC}=0.44$, and $\kappa \mathrm{BC}=0.39$, but some strong biases exist $(-0.35,-0.05$, and 0.27$)$. Thus, in the weak agreement between $\mathrm{A}$ and $\mathrm{B}$, pathologist C's own vote often determined the majority result. Prediction would be much harder for an independent observer.

Conclusions: We have trained a computer to recognize mitotic figures, using the pathologists' consensus. The recall of this system for a high precision is within the bounds of the performance of the original pathologists, if they had to commit only to affrmative or negative decisions. Previous work has investigated agreement in mitotic grades, which are determined by the total number of mitotic figures detected. We have evaluated pathologist agreement on individual mitotic figure recognition, on an unprecedented number of figure samples. In so doing, we have exposed systematic differences in the kinds of patterns counted by different pathologists.

\section{References}

[1] J.P.A. Baak, E. Gudlaugsson, I. Skaland, L.H.R. Guo, J. Klos, T.H. Lende, H. Soiland, E.A.M. Janssen, and A. zur Hausen, Proliferation is the strongest prognosticator in node-negative breast

Table 1

Prediction of test figures where two pathologists agreed

\begin{tabular}{|c|c|c|c|c|c|c|}
\hline Observer & Majority label & Observer "Mitosis" & $\begin{array}{l}\text { Observer } \\
\text { "May be" }\end{array}$ & $\begin{array}{c}\text { Observer } \\
\text { "Not mitosis" }\end{array}$ & Lower bound agreement & Upper bound agreement \\
\hline \multirow[t]{2}{*}{ A } & Positive (726) & 658 & 65 & 3 & $90.6 \%$ & $99.6 \%$ \\
\hline & Negative (73) & 15 & 36 & 22 & $30.1 \%$ & $79.4 \%$ \\
\hline \multirow[t]{2}{*}{ B } & Positive (726) & 394 & 166 & 166 & $54.3 \%$ & $77.1 \%$ \\
\hline & Negative (73) & 0 & 0 & 73 & $100.0 \%$ & $100.0 \%$ \\
\hline \multirow[t]{2}{*}{$\mathrm{C}$} & Positive (726) & 720 & 4 & 2 & $99.2 \%$ & $99.7 \%$ \\
\hline & Negative (73) & 2 & 2 & 69 & $94.5 \%$ & $97.3 \%$ \\
\hline \multirow[t]{2}{*}{ Machine } & Positive (726) & 462 & 0 & 264 & $63.6 \%$ & $63.6 \%$ \\
\hline & Negative (73) & 1 & 0 & 72 & $98.6 \%$ & $98.6 \%$ \\
\hline
\end{tabular}


cancer: Significance, error sources, alternatives, and comparison with molecular prognostic markers, Breast Cancer Res Treat 115 (2009), 241254.

[2] H.J. Bloom and W.W. Richardson. Histological grading and prognosis in breast cancer. A study of 1409 cases of which 359 have been followed for 15 years. Br. J. Cancer 11 (1957), 359-377.

[3] J.S. Meyer, C. Alvarez, C. Milikowski, N. Olson, I. Russo, J. Russo, A. Glass, B.A. Zehnbauer, K. Lister, and R. Parwaresch. Breast carcinoma malignancy grading by Bloom-Richardson system vs proliferation index: Reproducibility of grade and advantages of proliferation index. Modern Pathology 18 (2005), 1067-1078.

[4] P. Robbins, S. Pinder, N. de Klerk, H. Dawkins, J. Harvey, G. Sterrett, I. Ellis, and C. Elston. Histological grading of breast carcinomas: A study of interobserver agreement. Hum Pathol 26 (1995), 873-879.

\section{Application of digital pathology to create pathology assisting software to standardize the diagnosis of interstitial pneumonia}

Junya Fukuoka, Manami Saito, Tomonori Tanaka, Kyoko Otani, Takashi Hori and Sayuri Nunomura

Background: Pathological diagnosis in the field of rare disease usually requires special education and training. Especially for the many non-neoplastic diseases, the pathological diagnoses can only be judged by combination of non-specific findings. Usually, the order of importance and/or specificity for each finding is not well described in the textbook, and experience of cases only can give pathologists senses of judgment in each field. Considering the amount of complexity and increase in number of biopsy for upcoming personalized medicine and the shortage of pathologists throughout the world, pathology assisting system to standardize pathological diagnosis is highly needed. The area of interstitial lung disease (ILD) is one of the most complex fields, and the accurate distinction of usual interstitial pneumonia (UIP), highly lethal disease, from other types of ILD is a critical but difficult role for the surgical pathologists.

Material and methods: We have first examined the agreement of diagnosis for the 20 cases of interstitial lung disease among 29 pathologists including experts and non-experts for pulmonary pathology. Second, to know the importance of findings which leads to the diagnosis of UIP, a system to record evaluating process of each finding before calling a specific pathological diagnosis was created using whole slide scanning images (WSI). In short, WSI of the same 20 cases were analyzed by four pulmonary pathologists. The software was designated to evaluate 17 queries regarding histological findings using WSI of the 20 cases before reaching to the final diagnosis. Region of interests (ROI) images for positively scored findings were all captured for further discussion. Scoring data in each finding given by four panelists were analyzed statistically using linear regression analysis, and the skewness coefficients (SC) for each finding toward the diagnosis of UIP were calculated.

Results and discussion: The generalized kappa coefficient among 29 panelists was confirmed to be low as 0.21 . If it was limited to pulmonary experts, the kappa value was still not greatest (0.41). Among recorded 17 findings by the software, 6 showed significant positive/ negative SC. The 6 findings include levels of scarring and architectural destruction. Currently, we are creating the software that assists pathological diagnosis for general surgical pathologists using the 6 findings. The utility of the software and digital pathology for the routine clinic will be discussed in the session as well.

\section{Possibilities of digital navigation systems for pathology diagnosis}

\section{Yasunari Tsuchihashi}

Department of Clinical Pathology Research, Louis Pasteur Centre for Medical Research, Kyoto, Japan

Background: Pathology diagnosis is a series of informational processes starting from a biopsy material or from an extirpated surgical material to finally reach one sentence of diagnosis. The diagnostic processes are known to harbor pitfalls for human errors and may include monotonous laborious processes. In the age of digital pathology we may be able to create various navigational systems for ideal pathology diagnosis to ensure a correct diagnosis or enhance diagnostic efficiency. In the present study possibilities of digital navigations in pathology diagnosis are investigated.

Methods: Pathology diagnostic work flows of pathology laboratories of the three major regional hospitals in Kyoto, Japan were investigated in view of potential pitfalls for human errors and efficiency of the 
diagnostic works. Possible digital pathology navigational systems for idealistic pathology diagnosis were also extensively investigated.

Results: Automatic review of such basic patient records as name, age, sex, clinical records, etc, were possible in the routine laboratory diagnosis of the regional hospitals but not in their telepathology diagnostic systems. The lack of the automatic review of the information constituted a potential for human error. Review of digital radiology images of a patient was not available in two of the three regional hospitals owing to limited function of their hospital information system (HIS). Comparison of digital radiology images to digital macroscopic pathology images is functionally possible but still not in common use. Comparison of digital macroscopic image at a specific point of tissue sampling of an extirpated organ to its specific microscopic image is experimentally possible but currently not in practical use. Automatic detection of fragmented tissues on a slide and their realignment for a certain diagnostic purpose as modified digital images is experimentally possible but still not in practical use. Automatic detection of a certain tumour area in a given digital microscopic image is now experimentally possible but still not in practical use.

Conclusions: It is clear that there are a lot of possibilities for digital navigational systems for pathology diagnosis to enhance diagnostic efficiency or to ensure correct diagnosis. We need navigations for idealistic pathology diagnostic processes and digital pathology will provide the solution.

\section{Evaluation of Ultra High Speed WSI viewing system}

Yukako Yagi ${ }^{1}$, Hiroshi Kyusojin ${ }^{2}$, Shigeatsu Yoshioka $^{2}$, Maristela Onozato ${ }^{1}$, Eugene J. Mark ${ }^{1}$, Matthew P. Frosch ${ }^{1}$ and David N. Louis ${ }^{1}$

${ }^{1}$ Massachusetts General Hospital, Boston, MA, USA ${ }^{2}$ Sony Corporation, Tokyo, Japan

Context: The ability to digitize histopathology slides automatically, rapidly and with high resolution has been pursued by numerous investigators around the world. One of the goals is to implement into the clinical practice in Pathology. One of the pending issues is the "speed" of the entire process. The scanning time is faster and the performance of the scanner is more stable, however, the viewing speed is still not satisfactory for most users. We have evaluated a new whole slide imaging viewing station focusing on accessing speed, not only the speed to manipulate the Whole Slide Images (WSI) but also the speed to complete the tasks in many situations such as primary diagnosis, conference, teaching, etc.

Design: PlayStation ${ }^{\circledR} 3$ and wireless controllers were adapted to our study because it can manipulate huge graphic data without any stress locally and also over the network. We have evaluated the system at three situations at the Department of Pathology of MGH. 1. Simulation of signing-out cases, 2 Discussion tool at a consensus conference, 3 . Usage at a teaching course. Prior to the trials, we analyzed the required functions of the Graphic-User Interface (GUI) and Human Interface for each purpose and slightly different GUI was made to fit each purpose ideally.

Results: The system had a remarkable performance. The points of evaluation were 1. user-friendliness, 2. less frustration, 3 fit to purpose. Pathologists were able to use the system comfortably after $0-15$ min training. Pathologists who played the game regularly at home required 0-3 minutes training. Pathologists who never played the game required $10-15$ min training session. There were no complaints regarding the speed. Most pathologists were satisfied with the functionality, usability and speed of the system. The most difficult situation was to simulate signing-out of cases.

Conclusions: The preliminary results of our Ultra High Speed WSI Viewing System were promising. The speed of viewing the images demonstrated the possibility to use WSI in the daily practice in a near future. We continue to evaluate the system for further improvements.

Acknowledgment: The authors thank Department of Pathology, Massachusetts General Hospital, Boston, MA, USA for the collaboration.

\section{Ultra high speed wsi viewing system}

Shigeatsu Yoshioka ${ }^{1}$, Yukako Yagi ${ }^{2}$, Naoki Tagami ${ }^{1}$, Hiroshi Kyusojin $^{1}$, Masashi Kimoto ${ }^{1}$, Yoichi Mizutani $^{1}$, Kiyoshi Osato ${ }^{1}$ and Hiroaki Yada ${ }^{1}$

${ }^{1}$ Sony Corporation, Tokyo, Japan

${ }^{2}$ Harvard Medical School and Massachusetts General Hospital, Boston, MA, USA

Background: Over the years, we have gained real-time processing technology for minimum latency operation 
in game area. Among its many perceived applications to different areas, this technology will be especially useful for digital pathology where it will allow the handling of huge image data without delay.

Methods: In this work, we applied PS ${ }^{\circledR}$ to digital pathology viewer for whole-slide images to evaluate the potential benefit. A wide variety of images were used for the evaluation of viewing speed and usability - surgical biopsy, needle biopsy, mucosal biopsy, polypectomy, cytology, H\&E stain, IHC stain, etc. In terms of data size, the largest one is about $2 \mathrm{~GB}$ at $1 / 10$ compression rate.

Results: Due to the effect of high-speed processor and cache hit rate improvement using movement prediction prefetch, most of operations are reflected by the display in $1 / 60 \mathrm{~s}$. Even in the case of cache miss, browsing latency is several times shorter than existing viewers. Moreover, applying game controller brings several advantages including tireless panning and simultaneous multiuser operation.

Conclusions: By using PS3 ${ }^{\circledR}$, our viewer eliminated the negative effects of pathology digitalization and derived the benefits. It can be the tool that improves the efficiency of daily operations.

Acknowledgment: The authors thank Department of Pathology, Massachusetts General Hospital, Boston, MA, USA for the collaboration.

\section{Spectral sensing method for practical use}

Yasuhiro Fukunaga, Saori Shimizu, Kensuke Ishii and Kosei Tamiya

Olympus, Inc., Japan

Background: Digital color correction technology for image analysis and/or diagnosis is developed. Prior researchers have used monochrome camera with VariSpecTM tunable imaging filter or field/frame sequential color camera. However, it takes a long time to capture a number of spectral images in series.

Methods: The objective of this work is to develop a spectral sensing method for practical use. A light beam is divided into two by half mirror. One light beam is for RGB camera and the other is for spectral sensing unit. The spectrum can be sensed at the same time of capturing image by RGB camera. An area of spectral sensing is smaller than image and larger than picture element of RGB camera.
Results: We applied the spectral sensing unit for color correction of stained tissue images. 20 spectrums of specimens were enough for color correction of whole slide image. Each spectrum was taken within $235 \mathrm{msec}$. We found it corrected the color variation of H\&E images successfully.

Conclusions: We proposed a spectral sensing method suitable for digital color correction for image analysis. We will evaluate effectiveness of the proposed method for diagnosis with Whole Slide Imaging system.

\section{Color standardization system implementing estimation method for absorption spectra of dye}

Shinsuke Tani, Kensuke Ishi and Munenori Fukunishi

Olympus, Inc., Japan

Background: Color standardization is indispensable for implementing "tele-pathology" and "computer assisted diagnosis" for clinical uses, because the color variations affect the performance of digital image analysis as well as confusion of pathologists. These color variations are caused by not only device conditions but also staining methods, agent, environment, and so on. We developed color standardization based on multi-spectral techniques. In our previous method, however, the workflow is not practical for pathologists, because it is necessary for them to measure the absorption spectra using training samples stained by pure dye. We propose the advanced method to improve the accuracy and flexibility of color standardization without training samples.

Method: We develop the method with a multi-spectral sensor module and software installed into a digital microscope system. The algorithm is consists of the following procedure: 1 ) we acquire both the RGB image and the multi-spectral signals from multiple points on the specimens. 2) We estimate the absorption spectra of dye from the multi-spectral signals based on statistical method. 3) We implement color standardization using the estimated results.

Results: Without any training sample, we successfully estimate the absorption spectra of dye and standardize colors of H\&E images. We evaluate the effectiveness of our proposed method at the viewpoint of image feature detection for digital diagnostic assistance. 
Conclusion: For color standardization, we proposed an advanced method suitable for pathological workflow. We successfully standardized the color variation of H\&E images by applying the method to digital microscope systems.

\section{Whole slide imaging and expert analysis in adenocarcinoma of the prostate}

\section{Philippe Camparo}

Service d'Anatomie et Cytologie Pathologique, Hopital Foch, Suresnes, Paris, France

\section{Digital pathology (DP) in Asia}

\section{Robert Y. Osamura}

International University of Health and Welfare (IUHW), Center for Diagnostic Pathology, IUHW Mita Hospital, Minato-ku, Tokyo, Japan

General concept: Digital Pathology (DP) has been globally activated by whole slide imaging (WSI). The virtual microscopes (VM) with good quality of images are now available and they have been very competitive recently, particularly the speed of scanning at $\times 40$. When we deal with inter-continental or international DP, the practical application on histopathology and cytopathology includes consultation, frozen section rapid diagnosis, daily surgical pathology, daily cytopathology, automated image analysis and education in diagnostic pathology. As communication tools, IP-VPN and DICOM are the key issues for the efficient image transfer and the security-protected and widely usable DP.

DP in Asia: Background: We have experienced that the internet-based communication of digitized pathology images was satisfactory for the global DP between Japan (IUHW) and Asia, USA and Europe. We also successfully attempted the remote-pathological diagnosis using WSI and a satellite Kizuna which was supported by Japan Aerospace Exploration Agency (JAXA). This could be applied to DP at global basis. Currently the DP in Asia is still limited to some countries such as USA-China, USA-Singapore, Japan-China and a few others, but the Asian market has attracted international manufactures to develop DP networks.

DP in Asia: IUHW project: With the aid from the Ministry of Economy, Trade and Industry, IUHW is planning to explore the possibility to establish AsiaJapan DP networks for the diagnostic pathology. Our research focuses on the need of WSI scanners and DP networks in Asian regions using IP-VPN and DICOM system. Our project is composed of two researches, (1) local Asian need of DP in diagnostic pathology with communication to overseas hospital such as IUHW, (2) experiment with image transfer to IUHW Mita Hospital by setting up a image scanner in the Asia which is connected with IP-VPN. And we have found that the key issues here emphasize English as a common language, efficient image transfer, common diagnostic terminology in pathology.

Conclusions: In order to make the standardized and high quality diagnostic pathology available in Asia regions, DP networks are expected to serve as inevitable tools.

\section{Digital Image Analysis in pathology: Benefits and obligations}

\section{Arvydas Laurinavicius}

National Centre of Pathology, Vilnius, Lithuania

\section{Webmicroscope as a computer-based system for practical examination of dental students in oral pathology}

Janusz Szymas ${ }^{1}$, Mikael Lundin² ${ }^{2}$ and Johan Lundin ${ }^{2}$

${ }^{1}$ Department of Clinical Pathology, University of

Medical Sciences, Poznan, Poland

${ }^{2}$ Institute for Molecular Medicine Finland FIMM,

University of Helsinki, Helsinki, Finland

Before our experiment in 2006, virtual microscopy had not been used in an examination setting. On the other hand, pathology is a highly visual discipline. Therefore, the efficiency of teaching with the use of virtual slides should be tested at practical examinations with the direct and unlimited access to digitized slides. In order to be able to implement this system, we needed to find a solution of a difficult technical issue: providing a secure access to local teaching resources from a teaching laboratory through a general internet access without any noise or interruptions.

Methods: We decided to use two servers at two different locations (Poznan, Poland and Helsinki, Finland) and a stable, mature technology of a secure 
browser. As our examination system involves advanced HTML features, a web browser that can handle frames, javascript and cookies is required for viewing digitized histological slide and answering multiple choice questions at the same time. An online practical examination was evaluated based on students' results as well as a questionnaire.

Results: During the last five years, an overall 92\% concordance rate has been achieved on practical examination based on 50 virtual slides linked to 50 multiple choice questions per student. These results were independent from the number of slides used during the practicals in the last six years (50-112 slides). All students preferred the on-line examination and all felt that the quality of digitized slides was superior to that of "classical" slides and helped to make an accurate diagnosis (rating 9.5 on a $1-10$ scale). No technical disturbances have occurred so far.

Conclusions: The type of examination we propose is the most appropriate for pathology courses. The current formal evaluations of our examination system have provided us with a number of insights into the quality and effectiveness of digitized slides as a tool for learning and assessment. Current technologies are feasible for examination through broadband Internet.

\section{Virtual microscopy - a new tool in quality control and assurance of automated laboratory processes}

\author{
Gian Kayser ${ }^{1}$, Martin Werner ${ }^{1}$ and Klaus Kayser ${ }^{2}$ \\ ${ }^{1}$ Institute of Pathology, University Hospital Freiburg, \\ Freiburg, Germany \\ ${ }^{2}$ Institute of Pathology, Charite, Berlin, Germany
}

Context: Virtual microscopy is on the verge to take a new step: integration into routine histological diagnostics. In parallel with the increments in scanner speed, optical resolution and image quality, quantitative pathology and morphometry experience a renaissance.

Theory and present stage: Automated algorithms for tissue detection and in a semi-automated way also for immunohistochemical analyses have been introduced. Many of the latter are commercially available. In terms of quality management, control and assurance the pressure on histological laboratories is rising during the past decade. Most institutes in Germany have introduced standard operating procedures for laboratory processes to guarantee constant quality. Automation of embedding and staining procedures are to assist in these issues.

Results: Despite all efforts loss of tissue, inadequate tissue embedding, tissue cutting, or poor staining results do still occur. Analyzing the workflow of surgical pathological laboratories and implementation of virtual microscopy in combination with automated image analysis opens opportunities for process improvement, and, in addition, in the field of quality management, assessment, and certification.

Perspectives: Herein, we propose new concepts in virtual microscopy for quality control and assurance in histopathology.

\section{Using computerized workflow simulations to assess the feasibility of Whole Slide Imaging full adoption in a high volume histology laboratory}

David S. McClintock, Roy E. Lee and John R. Gilbertson

Department of Pathology, Masschusetts General Hospital, Boston, MA, USA

Context: WSI has been touted by many as the future of pathology, with estimates of full adoption of WSI occurring sometime in the next 5-15 years. While the technology surrounding the scanning of a glass slide has improved tremendously over the past decade, (average scan times of $60-90 \mathrm{sec}, 15 \times 15 \mathrm{~mm}$ at $40 \times$ ), there has been little consideration of how WSI will be implemented and subsequently affect the workflow of histology laboratories.

Design: Workflow process data for the histology laboratory at Massachusetts General Hospital was collected regarding: 1) process steps in the workflow, 2) available equipment and personnel resources, and 3) estimated volume of daily assets (blocks and slides). Using this information, a process model was developed using the business process management software iGrafx Process (Corel) and a computer simulation of the current histology laboratory was created. Histology laboratory simulations were run without and then with the presence of a WSI robot in the workflow and the effects on total process time and individual process steps were analyzed.

Results: Graphical representations of the simulations are shown in Fig. 1. Simulation data without and 
with the presence of WSI robots is shown in Fig. 2. Total process time increased 16.6 fold with the presence of one WSI robot. To minimize the effect of the WSI robot on workflow, at least 10 WSI were needed (without making major changes in workflow).

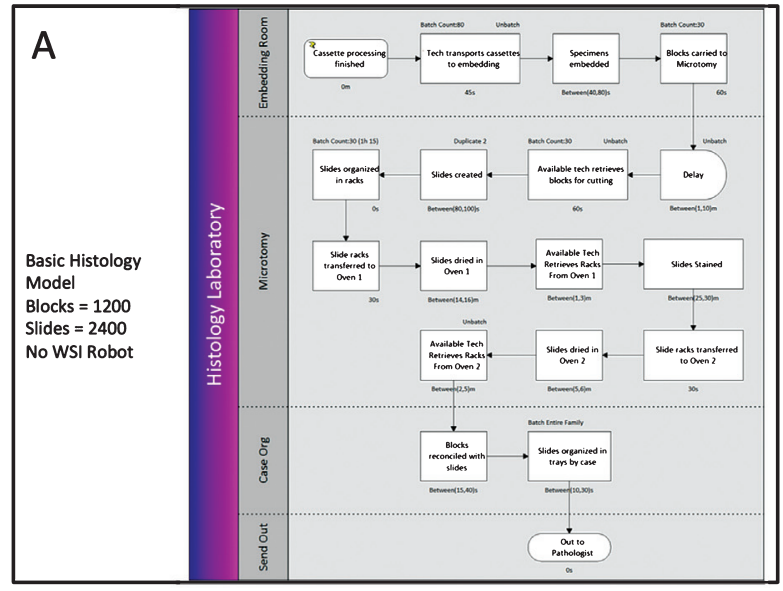

Conclusions: The effects of placing a WSI robot into the current setup of a histology laboratory with the intent of full adoption is unfeasible without 1) making major changes to the workflow of the histology laboratory, or 2) adding many WSI robots (approximately 1

Fig. 1. (A) Histology simulation models without WSI robot and (B) with WSI robot.

A

Elapsed Time (Hours)

\begin{tabular}{|r|r|r|r|r|r|r|r|r|r|r|r|r|}
\hline $\operatorname{Sim} \# 1$ & $\operatorname{Sim} \# 2$ & $\operatorname{Sim} \# 3$ & $\operatorname{Sim} \# 4$ & $\operatorname{Sim} \# 5$ & $\operatorname{Sim} \# 6$ & $\operatorname{Sim} \# 7$ & $\operatorname{Sim} \# 8$ & $\operatorname{Sim} \# 9$ & $\operatorname{Sim} \# 10$ & $\operatorname{Sim} \# 11$ & $\operatorname{Sim} \# 12$ & $\operatorname{Sim} \# 13$ \\
\hline 10.40 & 172.27 & 59.50 & 37.91 & 30.38 & 25.85 & 16.33 & 14.18 & 12.57 & 11.31 & 10.45 & 10.43 & 10.43 \\
\hline
\end{tabular}

Sim \#1 = No WSI robot, Sims \#2 - 13 = 1-12 WSI robot(s)

B

\begin{tabular}{|c|c|c|c|c|c|c|c|c|}
\hline \multicolumn{9}{|c|}{ Activity Statistics (Minutes) } \\
\hline \multicolumn{9}{|c|}{$\operatorname{Sim} * 1$} \\
\hline & Count & Avg Cycle & Avg Work & Avg Wait & Avg Res Wait & Avg Block & Avg Inact & Avg Sen \\
\hline Histology Laboratory/Embedding Room - Cassette processing finished & 1200 & 0.00 & 0.00 & 0.00 & 0.00 & 0.00 & 0.00 & 0.00 \\
\hline Histology Laboratory/Embedding Room - Specimens embedded & 1200 & 73.26 & 1.00 & 72.25 & 72.25 & 0.00 & 0.00 & 73.26 \\
\hline Histology Laboratory/Embedding Room - Tech transports cassettes to embedding & 1200 & 24.70 & 0.75 & 23.95 & 21.95 & 2.00 & 0.00 & 24.70 \\
\hline Histology Laboratory/Embedding Room - Blocks carried to Microtomy & 40 & 1.54 & 1.00 & 0.54 & 0.54 & 0.00 & 0.00 & 1.5 \\
\hline Histology Laboratory/Microtomy - Available tech retrieves blocks for cutting & 1200 & 1.11 & 1.00 & 0.11 & 0.11 & 0.00 & 0.00 & 1.1 \\
\hline Histology Laboratory/Microtomy - Slides created & 2400 & 2.69 & 1.50 & 1.19 & 1.19 & 0.00 & 0.00 & 2.65 \\
\hline Histology Laboratory/Microtomy - Slides organized in racks & 80 & 0.00 & 0.00 & 0.00 & 0.00 & 0.00 & 0.00 & 0.00 \\
\hline Histology Laboratory/Microtomy - Slide racks transferred to Oven 1 & 80 & 1.07 & 0.50 & 0.57 & 0.57 & 0.00 & 0.00 & 1.07 \\
\hline Histology Laboratory/Microtomy - Slides dried in Oven 1 & 80 & 15.02 & 15.02 & 0.00 & 0.00 & 0.00 & 000 & 1502 \\
\hline Histology Laboratory/Microtomy - Available Tech Retrieves Racks From Oven 1 & 80 & 2.71 & 2.06 & 0.65 & 0.65 & 0.00 & 0.00 & 27 \\
\hline Histology Laboratory/Microtomy - Slides Stained & 80 & 27.52 & 27.52 & 0.00 & 0.00 & 0.00 & 0.00 & 27.52 \\
\hline Histology Laboratory/Microtomy - Slides dried in Oven 2 & 80 & 5.45 & 5.45 & 0.00 & 0.00 & 0.00 & 0.00 & 5.45 \\
\hline Histology Laboratory/Microtomy - Delay & 1200 & 5.22 & 5.22 & 0.00 & 0.00 & 0.00 & 0.00 & 5.22 \\
\hline Histology Laboratory/Microtomy - Slide racks transferred to Oven 2 & 80 & 1.04 & 0.50 & 0.54 & 0.54 & 0.00 & 0.00 & 1.0 \\
\hline Histology Laboratory/Microtomy - Available Tech Retrieves Racks From Oven 2 & 2400 & 3.93 & 3.48 & 0.45 & 0.45 & 0.00 & 0.00 & 3.93 \\
\hline Histology Laboratory/Case Org - Blocks reconciled with slides & 2400 & 72.98 & 0.46 & 72.52 & 72.52 & 0.00 & 0.00 & 72.98 \\
\hline Histology Laboratory/Case Org - Slides organized in trays by case & 1200 & 0.33 & 0.33 & 0.00 & 0.00 & 0.00 & 0.00 & 0.33 \\
\hline \multicolumn{9}{|l|}{ Histology Laboratory/Case Org - Slides scanned in WSI robot } \\
\hline Histology Laboratory/Send Out - Out to Pathologist & 2400 & 0.00 & 0.00 & 0.00 & 0.00 & 0.00 & 0.00 & 0.00 \\
\hline
\end{tabular}

Fig. 2. (A) Simulation data, Total Process Time, (B) Individual Process Statistics Without WSI Robot, (C) Individual Process Statistics With One WSI Robot. 


\begin{tabular}{|c|c|c|c|c|c|c|c|c|}
\hline \multicolumn{9}{|c|}{ Activity Statistics (Minutes) } \\
\hline \multicolumn{9}{|c|}{ Sim \#2 } \\
\hline & Count & Avg Cycle & Avg Work & Avg Wait & Avg Res Wait & Avg Block & Avg Inact & Avg Serv \\
\hline Histology Laboratory/Embedding Room - Cassette processing finished & 1200 & 0.00 & 0.00 & 0.00 & 0.00 & 0.00 & 0.00 & 0.00 \\
\hline Histology Laboratory/Embedding Room - Specimens embedded & 1200 & 73.26 & 1.00 & 72.25 & 72.25 & 0.00 & 0.00 & 73.26 \\
\hline Histology Laboratory/Embedding Room - Tech transports cassettes to embedding & 1200 & 24.70 & 0.75 & 23.95 & 21.95 & 2.00 & 0.00 & 24.70 \\
\hline Histology Laboratory/Embedding Room - Blocks carried to Microtomy & 40 & 1.54 & 1.00 & 0.54 & 0.54 & 0.00 & 0.00 & 1.54 \\
\hline Histology Laboratory/Microtomy - Available tech retrieves blocks for cutting & 1200 & 1.11 & 1.00 & 0.11 & 0.11 & 0.00 & 0.00 & 1.11 \\
\hline Histology Laboratory/Microtomy - Slides created & 2400 & 2.69 & 1.50 & 1.19 & 1.19 & 0.00 & 0.00 & 2.69 \\
\hline Histology Laboratory/Microtomy - Slides organized in racks & 80 & 0.00 & 0.00 & 0.00 & 0.00 & 0.00 & 0.00 & 0.00 \\
\hline Histology Laboratory/Microtomy - Slide racks transferred to Oven 1 & 80 & 1.07 & 0.50 & 0.57 & 0.57 & 0.00 & 0.00 & 1.07 \\
\hline Histology Laboratory/Microtomy - Slides dried in Oven 1 & 80 & 15.02 & 15.02 & 0.00 & 0.00 & 0.00 & 0.00 & 15.02 \\
\hline Histology Laboratory/Microtomy - Available Tech Retrieves Racks From Oven 1 & 80 & 2.71 & 2.06 & 0.65 & 0.65 & 0.00 & 0.00 & 2.71 \\
\hline Histology Laboratory/Microtomy - Slides Stained & 80 & 27.52 & 27.52 & 0.00 & 0.00 & 0.00 & 0.00 & 27.52 \\
\hline Histology Laboratory/Microtomy - Slides dried in Oven 2 & 80 & 5.45 & 5.45 & 0.00 & 0.00 & 0.00 & 0.00 & 5.45 \\
\hline Histology Laboratory/Microtomy - Delay & 1200 & 5.22 & 5.22 & 0.00 & 0.00 & 0.00 & 0.00 & 5.22 \\
\hline Histology Laboratory/Microtomy - Slide racks transferred to Oven 2 & 80 & 1.04 & 0.50 & 0.54 & 0.54 & 0.00 & 0.00 & 1.04 \\
\hline Histology Laboratory/Microtomy - Available Tech Retrieves Racks From Oven 2 & 2400 & 3.93 & 3.48 & 0.45 & 0.45 & 0.00 & 0.00 & 3.93 \\
\hline Histology Laboratory/Case Org - Blocks reconciled with slides & 2400 & 0.95 & 0.46 & 0.49 & 0.00 & 0.00 & 0.49 & 0.46 \\
\hline Histology Laboratory/Case Org - Slides organized in trays by case & 1200 & 0.33 & 0.33 & 0.00 & 0.00 & 0.00 & 0.00 & 0.33 \\
\hline Histology Laboratory/Case Org - Slides scanned in WSI robot & 2400 & 3511.55 & 2.26 & 3509.28 & 2525.07 & 0.00 & 984.21 & 2527.34 \\
\hline Histology Laboratory/Send Out - Out to Pathologist & 2400 & 0.00 & 0.00 & 0.00 & 0.00 & 0.00 & 0.00 & 0.00 \\
\hline
\end{tabular}

Fig. 2. Continued

WSI robot/240 slides scanned). These solutions, while possible with current technology, are not without significant costs that must be considered prior to the full adoption of whole slide imaging.

\section{The e-Pathologist Cancer Diagnosis Assistance System for gastric biopsy tissues}

Maki Ogura ${ }^{1,2}$, Akira Saito ${ }^{1}$, Hans Peter Gra $^{3}$, Eric Cosatto $^{3}$, Christopher Malon ${ }^{3}$, Atsushi Marugame ${ }^{1}$, Tomoharu Kiyuna ${ }^{1}$, Yoshiko Yamashita ${ }^{1}$, and Manabu Fukumoto ${ }^{2}$

${ }^{1}$ Innovative Service Solutions Division, NEC Corporation, USA

${ }^{2}$ Department of Pathology, Institute of Development, Aging and Cancer, Tohoku University, Miyagi,

Japan

${ }^{3}$ NEC Laboratories America, Inc, USA
Background: Recently, several vendors have developed slide scanners and counting systems for the automated analysis of immunohistochemically stained tissues. However there is no such a system for $\mathrm{H} \& \mathrm{E}$ stained tissue sections. This is very difficult since such tissues exhibit a complex mixture of overlapping malignant tumor cells, non-malignant cells and extracellular matrices. At NEC we have been made efforts to develop a Cancer Diagnosis Assistance System (CDAS), called e-Pathologist since 2001. In this study, we demonstrate here the e-Pathologist's performance for H\&E stained gastric biopsy tissues and describe an example of its practical use.

Methods: A total of 1,905 tissues, prepared for the validation of the system, were first diagnosed by two independent pathologists. Of these, 1805 were classified as benign and 100 tissues as malignant. The malignant tissues consist of 5 types of carcinoma, that is well differentiated, moderately differentiated and

Table 1

Detection ratios of the tissue samples as malignant

\begin{tabular}{lcll}
\hline Algorithm & & Nos. of cases & Ratio \\
\hline Malignant cases Total & ROI detection 1Qx Confirmation 40× Signet-ring cell detections & 93,100 91/93 B/9 99100 & $99 \%$ \\
False positive Total & ROI detection 10× Confiimation40x & 4S11S05 254 4S1 & $12.6 \%$ \\
& & 227,1 SO5 & \\
\hline
\end{tabular}


poorly differentiated adenocarcinoma, as well as mucinous carcinoma, and signet ring cell carcinoma.

Results: A pathological diagnosis with the system first starts analyzing an entire H\&E-stained tissue sections at low magnification $(10 \times)$ then proceeds to locating suspicious areas for cancer (regions of interest: ROIs). Then these ROIs are analyzed at high magnification $(40 \times)$ to confirm the initially suspicious region. The analysis was performed on two major components. One was evaluation of structural abnormalities, as well as nuclear density, nuclear size and shape alterations, indices to classify adenocarcinomas into well, moderately and poorly differentiated types. The second was more specific analysis for gastric cancer, that is, searches for occurrences of signet ring cells. All processes of the system are summarized in Fig. 1. Overall, the system correctly diagnosed 99 out of the 100 positive samples and 1578 out of the 1805 negative samples. One out of 100 malignant cases was a false negative $(1.0 \%)$, and 227 out of 1805 benign cases were false positive (12.6\%). Details are given in Table 1.

Conclusions: In this study, we described a novel computerized analysis system, the CDAS e-Pathologist, and a large-scale test demonstrated its competence with very low rate of misdetections. This system was delivered to a clinical examination company in Japan, and has been performed the analysis of more than 50,000 slides for quality control and quality assurance of pathological diagnosis. In cooperation with Massachusetts General Hospital we started developing such an analysis system for breast cancer and prostate cancer. The plan is to adapt this system to various kinds of cancer in the near future.

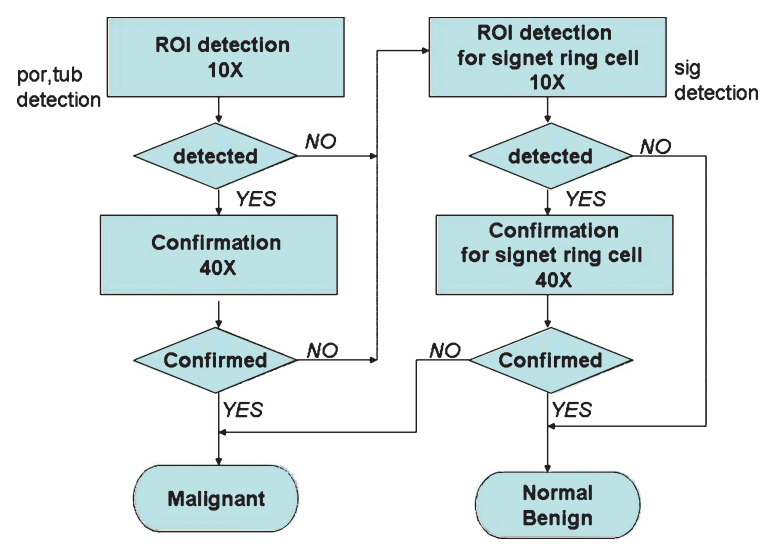

Fig. 1 Construction of the system.

\section{A comparison of digitized frozen section and smear preparations for intraoperative neurotelepathology}

\author{
Peter V. Gould, and Stephan Saikali
}

Service d'Anatomopathologie et de Cytologie, Centre hospitalier affilié universitaire de Québec, Hôpital de l'Enfant-Jésus, Québec, Canada

Background: Intraoperative consultations in europathology are often assessed by smear preparations rather than by frozen sections. Both techniques are standard practice for light microscopic examination on site, but there is little data comparing these techniques in a telepathology setting.

Methods: Thirty cases of brain tumours submitted for intraoperative consultation at our institution between July and December 2010 were identified in which both frozen section and tissue smear preparations were available for digitization at $20 \times$ magnification. Slides were digitized using a Hamamatsu Nanozoomer $2.0 \mathrm{HT}$ whole slide scanner, and resulting digital images were visualized at $1680 \times 1050$ pixel resolution with NDP.view software.

Results: The original intraoperative diagnosis was concordant with the sign out diagnosis in 29/30 cases; one tumeur was initially interpreted as a high grade glioma but proved to be a lymphoma at sign out.

Digitized frozen section slides were sufficient for diagnosis at $10 \times$ magnification in $27 / 30$ cases. Digitized tissue smears were sufficient for diagnosis at $10 \times$ magnification in $28 / 30$ cases. In two cases tumour was present on the tissue smear but not the frozen section (one case of recurrent astrocytoma, one case of meningeal carcinomatosis). In one case of lymphoma, tumour was present on frozen section only. These discrepancies were attributed to tissue sampling rather than image quality.

Examination of digitized slides at higher magnfication $(20 \times)$ permitted confirmation of mitoses and Rosenthal fibers on tissue smear preparations, but did not change the primary diagnosis. Intra-slide variations in tissue thickness on smear preparations led to variable loss of focus in digitized images, but did not affect image quality in thinner areas of the smear or impede diagnosis.

Conclusion: Digitized tissue smears are suitable for intraoperative neurotelepathology and provide comparable information to digitized frozen sections at medium power magnification. 


\section{Histopathologic patterns of nervous system tumors based on computer vision methods and whole slide imaging (WSI)}

Slawomir Walkowski and Janusz Szymas

University of Medical Sciences, Poznan, Poland

Background: Making an automatic diagnosis based on virtual slides, which are normally viewed by pathologists, is a big challenge. Determining whether a case belongs to a single class, representing a specific disease, could be the first step in the diagnosing process. In this work we focus on WHO Classification of Tumours of the Central Nervous System. We try to design a method which allows to automatically distinguish virtual slides which contain histopathologic patterns characteristic for glioblastoma - pseudopalisading necrosis and discriminate cases with neurinoma (schwannoma), which contain similar structures palisading (Verocay bodies).

Methods: Our method is based on creating a pattern with a set of features which can describe pseudopalisading necrosis and distinguish it from palisades. These parameters are initially used in image segmentation to extract nuclei on the slide, find necrosis areas and locate regions with high density and specific mutual arrangement of nuclei. Structural analysis of boundaries formed by these regions is done for final classification.

Results and conclusions: Described method is tested on a set of over a dozen virtual slides, captured using robotic Microscope Axioplan 2e (ZEISS) with objective Planapo $20 \times / 0,75$, equipped with Camera Axiocam HRc (ZEISS). The scanning process produced $1300 \times 1030$ tiles, with $15 \%$ overlap, which were stitched into single images. Test results show capabilities of the proposed method.

\section{How to introduce virtual microscopy (VM) in routine diagnostic pathology: Constraints, ideas, and solutions}

\author{
Klaus Kayser ${ }^{1}$, Stephan Borkenfeld ${ }^{2}$ and Gian \\ Kayser $^{3}$ \\ ${ }^{1}$ Charite, University of Berlin, Berlin, Germany \\ ${ }^{2}$ IAT, Heidelberg, Germany \\ ${ }^{3}$ Institute of Pathology, University of Freiburg, \\ Freiburg, Germany
}

Context: Virtual microscopy which is the diagnostic work with digitized microscopic images in tissue based diagnosis is in its childhood in being implemented in routine diagnosis. Until today, only a few pathology institutions take use of this new technology, although it is available since several years. Why?

Design: Virtual microscopy requires a new workflow organisation in the pathologist's diagnostic procedure. At a first view, the laboratory workflow seems to remain untouched to a high degree. However, the used laboratory information system (LIS), which is commonly built in a hierarchic order, has to be adjusted at its highest levels, i.e., diagnosis statement, quality evaluation, submission to the clinician (hospital information system), and feedback to the laboratory. Therefore, the laboratory's workflow is involved at all levels too, and the LIS has to be changed or adjusted to the requirements of VM. VM systems are usually equipped with a viewer that mimics the viewing of a conventional microscope, and do not offer access to sensitive nodes of the LIS. Similar, LIS are usually closed and fixed systems because of data security and certification demands. Thus, VM systems have to possess communication access at different LIS levels together with steering commands for the LIS in close association with the diagnostic quality and efficiency (for example demands for additional stains, immunohistochemical or quantitative image methods, etc.), as well as expert consultation, or panel discussion.

Results: An implementation of an open and active LIS - VM management system could significantly promote the introduction of VM into routine diagnostic surgical pathology. The management system has to coordinate and translate the demands of VM to LIS (and vice versa), and to assure the communication with HIS. Mandatory features include streaming of the laboratory workflow, feedback commands to LIS, as well as regulation of temporary priority levels.

Conclusion: A successful implementation of VM systems in routine tissue-based diagnosis requires communicative management systems as long as VM is considered to be a "stand alone system" that just mimics a conventional microscope. 
Molecular cytology core facility Efforts to accelerate the research process

K. Manova, M. Turkekul, Y. Romin, N. Fan, S. Oh, A. Baldys, S. Fujisawa and A. Barlas

Memorial Sloan Kettering Cancer Center, New York, $N Y, U S A$

Molecular Cytology Core Facility (MCCF) provides consultations and assistance in the execution of technologies for in situ localization and detection of molecular markers of cell metabolism and intercellular communications in normal and cancer cells and tissues. We serve the larger research community, including about 100 laboratories from MSKCC and laboratories from Weill Cornell Medical School and Rockefeller University. The experiments at the MCCF are either carried out by the MCCF staff or performed with our assistance by the researchers- users of MCCF.

Depending on the researcher needs, work on the project could start from tissue dissection/fixation, followed by preparation of the samples and execution of IHC, IF (single, double, triple and quadruple) or RNA in situ hybridization. The researchers could choose between being trained to perform the experiments manually or use our services for automated in situ detections.

Our antibody bank consists of more than 150 different antibodies. In addition, we have access to more than 400 antibodies, provided by our users. We maintain a digital record for the validation of the antibodies. Validation process involves preparation of proper positive and negative tissues. When control tissues are unavailable, especially for human samples, cultured cells are used. Cell suspensions are processed using tissue protocols. Mouse embryos are invaluable tissue controls, since genes expressed during the embryonic development are also expressed in the cancerous tissues. Automated IF experiments provide information about the special relationship of two or more molecules. Not only in cultured cells but also in tissue sections successful triple fluorescence detection has been achieved. Valuable data about simultaneous mRNA and protein detection in tissues has been generated.

Image acquisition is performed using the optical imaging systems at MCCF. The wide field microscopes are equipped for BF imaging, including DIC and polarized light, for DF and fluorescence imaging. Confocal microscopes (point scanning, line scanning and spinning disc) allow acquisition of $\mathrm{Z}$ stacks, automated and time lapse imaging. Multispectral imaging is available with confocal and with wide field microscopes. Digital scanners generate virtual bright field and fluorescence images.

Examples of MCCF research activities, including 2D and 3D analysis, particle tracking, 3D reconstructions and time lapse will be presented.

\section{Automatic analysis of virtual slides to help in the determination of well established prognostic parameters in breast carcinomas}

N. Elie $^{1}$, V. Becette ${ }^{2}$, B. Plancoulaine ${ }^{1}$, H. Pezeril ${ }^{2}$, M. Brecin' ${ }^{2}$, Y. Denoux ${ }^{2}$, J. Chasle 2 , C. Bor ${ }^{2}$ and P. Herlin ${ }^{1}$

${ }^{1}$ Unité "Histo-Imagerie Quantitative", GRECAN, EA 1772 de l'Université de Caen Basse-Normandie et IFR146 ICORE, CLCC François Baclesse, Caen, France

${ }^{2}$ Service d'Anatomo-Pathologie du CLCC François Baclesse, Caen, France

Background: Grading is a standard clinical practice for the diagnosis and prognosis of breast cancer. The internationally accepted system is that defined by Elston and Ellis. It is assessed by evaluating acinar formation, nuclear size/pleomorphism and mitotic activity. Pathologist-based visual evaluation on tissue slides is considered as the gold standard for tumor grading. However, the intra-and inter-observer reproducibility is still far from being perfect, due, in particular, to intra-tumor heterogeneity. Digital pathology on the virtual slides offers the opportunity to develop an automated and standardized grading system.

Methods: The study was conducted on 370 archival breast cancer specimens. The automated analysis was developed on a representative immunostained section of each breast tumor with an antibody targeting the mitotic figures (anti-phospho-Histone H3). Morphometric criteria were used on the whole section for the determination of glandular differentiation score. Nuclear pleomorphism was evaluated by taking advantage of the stereological volume weighted mean volume technique. Mitotic activity was automatically scored according to the method used by pathologists: identification and selection of the territory with the largest number of mitotic figures and counting mitotic figures in ten contiguous fields. The validation of this 
strategy was done at several levels: first, a quality control procedure based on stereology, to validate the choice of image analysis algorithms, then a study of concordance with the reference method (the grade defined by the pathologist on HE stained slide) and finally an evaluation of the prognostic value of the automated approach.

Results: After having selected the best image analysis strategy by reference with the quality control, the correlation between the grades obtained by the reference method and the automated system was significant $(r=0.58-p<0.05)$. The both grading systems had a prognostic significance: the grade provided by the pathologist $(p<10 \mathrm{e}-4)$ and obtained by the automated system $(p<0.0012)$.

Conclusions: Automated grading of breast carcinoma can be performed on virtual slides. In the future, such a validated tool could be helpful for diagnostic quality control as well as for central review of clinical trials.

\section{Automated quantification of liver steatosis by WSI based image analysis}

Roy Lee, Maristela Onozato, Nathan Shores, and Yukako Yagi

Pathology Information and Communication Technology (PICT) Laboratory, Department of Pathology, Massachusetts General Hospital, Boston, MA, USA

Introduction: Manual quantification of various histology findings such as steatosis quantification and $\mathrm{Ki}$ 67 indexing by pathologists are long known to suffer from wide inter- and intra-observer variability. Clinical ramifications of this variability can be substantial, as exemplified by the use of liver transplantation grafts that are already fatty. Methods to produce more consistent quantifications are thus needed. In the setting of whole slide imaging (WSI), we have developed an automated, image analysis algorithm that is trainable for various purposes. For this study, we used it to calculate total steatosis percentage for liver core biopsies, and compared the results against those from pathologists and also chemical lipid analysis.

Methods: Initially, 147 liver core biopsy WSI slides, each with non-alcoholic fatty liver disease (NAFLD), were used to train the algorithm, and then another 60 similar slides with NAFLD due to Hepatitis C and/or
Human Immunodeficiency Virus were used to refine the training. Quantification was based on total surface area involving steatosis and total surface area of the full core biopsy. The refined steatosis detection algorithm was then applied to 18 liver core biopsy WSI slides. A group of pathologists were also recruited to manually estimate steatosis percentage on these same slides, and physical recuts of each slide underwent chemical triglyceride treatment to objectively quantify the contained lipids on each of them.

Results: We found that there was a good correlation coefficient $(0.89)$ between the automated quantification and manual quantification by pathologists. Interestingly, the correlation coefficients between automated quantification/chemical analysis, and manual quantification/chemical analysis, were both lower, at 0.83 . In addition, in all cases, quantification values for steatosis performed manually by pathologists were larger than with the automated method.

Conclusion: Automated image analysis algorithms are needed to reduce in order to reduce intra- and inter-observer variability. Such algorithms would be an excellent application of whole slide imaging. Further studies to prove consistency with these automated methods as compared to manual methods (hypothesized to suffer from high inter- and intra- observer variability) are needed.

\section{Application of multi-scale filtering to cell nucleus detection with automated scale selection}

Hatice Cinar-Akakin ${ }^{1}$, Hui Kong ${ }^{1}$, Gerard

Lozanski $^{2}$ and Metin N. Gurcan ${ }^{1}$

${ }^{1}$ Department of Biomedical Informatics, The Ohio

State University, Columbus, OH, USA

${ }^{2}$ Department of Pathology, The Ohio State

University, Columbus, OH, USA

Background: The Ki67 immunohistochemical stain is used to evaluate proliferation fraction of cells in formalin fixed and paraffin embedded tissue. Ki67 based proliferation fraction is used as an important prognostic indicator in hematologic and solid tumor malignancies. Manual enumeration of the positively immunostained cells by the human readers is time-consuming and shows high intra- and inter-reader variability. Moreover, resolution of manual method of Ki67 scoring has poor resolution and cannot reproducibly detect 
Table 1

Statistics of number of detected cells for different levels of positive cell intensity

\begin{tabular}{lccccc}
\hline Level \& number of images & Level-1 (13) & Level-2 (13) & Level-3 (14) & Level-4 (12) & Level-5 (13) \\
\hline Average-Standard deviation & $960-117$ & $572-68$ & $273-45$ & $138-25$ & $60-10$ \\
Maximum & 1196 & 739 & 359 & 197 & 73 \\
Minimum & 833 & 456 & 205 & 108 & 43 \\
\hline
\end{tabular}

differences below $20 \%$. Therefore, our purpose is to develop and validate an automated tool that detects and counts positive cell nuclei. "Artificial lymph node tissues," comprised of mixed population of Raji and Jurkat cell lines mixed at different proportions at $20 \%$ interval starting from $100 \%$ Jurkat and ending with $100 \%$ Raji cells, were created to validate the system. We used TDT nuclear IHC staining as a surrogate for Ki67 stain. Hence, TDT images with varying number of positive cells were represented with Level-1 to Level-5, from highest to lowest number of cells respectively. In TDT immunostaining, potentially positive cells appear in hues of brown and the negative cells appear in hues of blue.

Methods: The first step is the identification of positive immunostaining in the TDT slides by brown color segmentation. We utilized specific channels of two color spaces, i.e., blue channel of RGB and saturation channel of HSV to train a Discriminant Analysis based classifier. After color segmentation, individual nuclei were detected by our novel algorithm that employs multi-scale Laplacian of Gaussian Filters with automatic scale selection.

Results: The automated nucleus detection and counting algorithm was tested on artificial lymph node tissue images. 65 ROI images of size $896 \times 1072$ pixels were cropped from the five whole slide images at randomly. The detection results are given in Table 1 .

Conclusions: Controlled experiments on artificially created tissues indicate that the developed system can accurately detect and count the positively stained cell nuclei. The system is automated including its scale parameter selection, which makes it possible to use it on cell images containing varying sizes of nuclei.

\section{Quantification of liver fibrosis by whole slide image analysis}

Tokiya Abe ${ }^{1}$, Ken Yamazaki ${ }^{1}$, Akinori Hashiguchi ${ }^{1}$, Hidetsugu Saito ${ }^{2,3}$ and Michiie Sakamoto ${ }^{1}$

${ }^{1}$ Department of Pathology, School of Medicine, Keio University, Tokyo, Japan

${ }^{2}$ Department of Internal Medicine, School of Medicine, Keio University, Tokyo, Japan

${ }^{3}$ Faculty of Pharmacy, Keio University, Tokyo, Japan

Background: Fibrosis progression and architectural changes in chronic liver disease are evaluated by liver biopsy using semi-quantitative numerical scores, which produce partially subjective evaluations. It is expected to establish quantification method of liver fibrosis in routine practice. As basic study, this paper measured a percentage of collagen by whole slide image analysis and investigated a relationship between the percentage, a histological score, and stiffness measured by Fibroscan ${ }^{\circledR}$.

Methods: Thirty-eight patients with liver damage, who received liver biopsy, were enrolled. Fibroscan ${ }^{\circledR}$ was performed at the same time as liver biopsy. Liver biopsy samples were evaluated histologically according to New Inuyama score for disease stage and digitized using NanoZoomer (Hamamatsu Photonics, Japan) for whole slide image analysis. In Elastica van Gieson (EVG) stained samples, collagen, elastin, nuclei, cytoplasm and background can usually be discerned by color contrast, so these components were defined as categories of classification in RGB color space. In this analysis, each pixel of whole slide image was segmented into five categories by classification based on their color distribution, which was implemented with a Gaussian classifier. Then, a percentage of collagen was measured. The color distribution of EVG tissue section was highly variable, so that the Gaussian classifier was designed for each EVG stained sample to discriminate precisely each category. When classifying each pixel with the Gaussian classifier, the 
mean vector and the covariance matrix were estimated from RGB signals of training data set.

Results: The percentage of collagen increased with worsening histological stage as expected. A Spearman's rank correlation coefficient between the percentage of collagen and histological score was 0.69 $(p<0.001)$. In the same way, a correlation coefficient between the percentage and Fibroscan ${ }^{\circledR}$ data was 0.66 $(p<0.001)$.

Conclusions: Our results showed that the five categories were appropriately segmented by whole slide image analysis of EVG stained samples and that the percentage of collagen correlated well with New Inuyama score and Fibroscan ${ }^{\circledR}$ data.

\section{Multifractal feature descriptor for histopathology}

Chamidu Atupelage ${ }^{1}$ Hiroshi Nagahashi ${ }^{2}$, Masahiro Yamaguchi $^{3}$, Michiie Sakamoto ${ }^{4}$ and Akinori Hashiguchi $^{4}$

\section{${ }^{1}$ Department of Computational Intelligence and Systems Science, Tokyo Institute of Technology, Tokyo, Japan \\ ${ }^{2}$ Imaging Science and Engineering Laboratory \\ ${ }^{3}$ Global Scientific Information and Computing Center, Tokyo Institute of Technology, Tokyo, Japan ${ }^{4}$ Department of Pathology, School of Medicine, Keio University, Tokyo, Japan}

Background: Histopathology is the study of microscopic anatomy of the body cells and widely using technique in cancer diagnosis. Pathologists investigate the biopsies using microscopic images for diagnosing diseases. However, this exploration is highly subjective. Therefore, it requires more concrete quantitative computational model to classify histopathologic images. This paper presents a multifractal analysis based feature description technique to discriminate different types of tissues in histopathologic images.

Methods: Multifractal analysis can utilize to obtain two kinds of texture descriptions; local singularities: $\alpha$ and global regularities: $F(\alpha)$. Different kinds of multifractal feature can be generated by alternating the characteristic function used in the initial computation. The proposed descriptor use six characteristic measures (4-intensity measures and 2-gradient measures) to turns the intensity domain of the image into 6-dimensional feature vector space; subsequently we utilized Texton dictionary technique with Support Vector Machine to distinguish the cancer and noncancer regions of histopathologic images of different body organs. We obtained two categories (Liver and Prostate) of annotated (cancer and non-cancer) histological data samples, which can visualize in eight magnification $(1.25 \times, 2.5 \times, 5.0 \times, 10 \times, 20 \times, 40 \times, 63 \times$, and $100 \times$ ) via a digital slider (Nano-Zoomer). The appearances of histological properties such as nuclei, nucleoli, cytoplasm, etc., are varied according to the magnification. Also the sizes of the cancer areas are differed in different biopsies. Therefore, we utilized the images of all magnifications in our experiments and generated the sample dataset by randomly collecting three sizes patches $(64 \times 64,128 \times 128$ and $256 \times 256$ pixels) in each whole slide image as equal amounts in the cancer and non-cancer regions. We employed these datasets with multifractal feature descriptor and two other steady feature descriptors including Gabor (16-filters were used) and GLOG (12filters were used) to perfume comparative experiment.

Results and conclusion: We measured the discrimination power as the correct classification rate (CCR) for each feature descriptor. Moreover, pathologists often use lower magnification of digital slider (ex: less than $40.00 \times$ ) for their diagnosis, because of the visual artifacts exhibited in larger magnifications. Results indicate the average CCRs of multifractal descriptor for both Liver and Prostate data are around $95 \%$ in lower magnifications. Also the proposed method outperforms other steady feature description techniques in all magnifications.

\section{A pathology imagery interpretability rating scale for virtual microscopy}

Peter Kragel and Bill Oliver

Department of Pathology and Laboratory Medicine, East Carolina University, Greenville, NC, USA

The National Imagery Interpretability Rating Scale (NIIRS) is a perceptual scale based on the ability of an observer to extract useful information (called "interpretability") from images. NIIRS criteria are developed at different levels and are based on a set of specific interpretation tasks. NIIRS has been the standard for intelligence imagery for thirty years, and is proven to be robust and useful in intelligence, military, and 
commercial applications. NIIRS is a perceptual scale; rather than being based on physical parameters such as edge sharpness, contrast, texture, etc., it defines quality in terms of the ability of an observer to extract useful information (called "interpretability"). NIIRS are generally sensor and domain specific, and normally have 10 levels. For example, NIIRS criteria for satellite imagery include:

Level 1: Distinguish between major land use classes (e.g., urban, agricultural, forest, water, barren);

Level 3: Detect individual houses in residential neighborhoods;

Level 5: Identify tents at recreational camping areas; and

Level 9: Identify individual barbs on a barbed wire fence.

The methodology for developing and validating NIIRS criteria is well-established, and can easily be modified to apply to light microscopy. To establish a Pathology Imagery Interpretability Rating Scale (PIIRS) for virtual microscopy, slides are scanned at a set magnification with additional magnification provided electronically. Specific criteria are developed at different levels, becoming more difficult to interpret as the levels increase. Examples include:

Level 1: Distinguish between broad architectural types (e.g., a large scar and myocardium in cardiac muscle);

Level 3: Identify Islet of Langerhans in an H\&E stained section of pancreas;

Level 5: Identify mitoses; and

Level 9: Identify cryptosporidium in an $\mathrm{H} \& \mathrm{E}$ stained section of small bowel.

A PIIRS for virtual microscopy would be of use in comparing and evaluating microscopy image acquisition and display systems, establishing criteria for technology acquisition, and evaluating changes in standard operating procedures. As the use of virtual microscopy increases, a standardized method of evaluating the interpretability of microscopic images, such as NIIRS, will be critical to assure the accuracy of histopathologic diagnoses.

\section{Development of image quality evaluation method for whole slide imaging}

Noriaki Hashimoto $^{1}$, Pinky A. Bautista ${ }^{2}$, Masahiro

Yamaguchi $^{3}$, Nagaaki Ohyama ${ }^{4}$ and Yukako Yagi ${ }^{2}$

${ }^{1}$ Interdisciplinary Graduate School of Science and Engineering, Tokyo Institute of Technology, Tokyo, Japan

${ }^{2}$ Department of Pathology, Harvard Medical School, MA, USA

${ }^{3}$ Global Scientific Information and Computing

Center, Tokyo Institute of Technology, Tokyo, Japan

${ }^{4}$ Imaging Science and Engineering Laboratory,

Tokyo Institute of Technology, Tokyo, Japan

Background: Recently, digital pathology has been rapidly developing, but there are still a number of problems; accurate color, high-quality image, standard format for storage and so on. It is very important that the whole slide imaging (WSI) scanner provides images of good quality for accurate digital diagnosis and image analysis in diagnostic support systems. Hence it is imperative to evaluate image quality of scanned slide. In this paper, we propose a method to evaluate the quality of scanned pathological slide images. Furthermore, we show that integrating such method to the whole slide scanning process will improve the overall image quality of scanned images.

Methods: The proposed method has to evaluate the image quality of target images without references. The proposed image quality evaluation method is based on image focus (sharpness) and noise. Using these evaluation indices, the definitive evaluation index is derived. First we performed an experiment to confirm the validity of the proposed evaluation method. Then, we applied the image-quality evaluation method to the entire whole slide image. The whole slide image, which was scanned in automatic mode, was divided into blocks, and image quality evaluation was applied to each block. The regions which had low image quality index were re-scanned and evaluated again.

Results: In the first experiment, we have confirmed that the evaluation index of the proposed method has the high correlation to subjective score through multiple regression analysis. In the application to WSI, the region which showed low quality was specified as the focus point and re-scanned after evaluating the image scanned by automatic mode. Evaluation on the image quality of the re-scanned regions shows 
noteworthy improvement. This illustrates that the overall quality of scanned images could be improved by incorporating image quality evaluation in the scanning process.

Conclusion: In this work we presented an image quality evaluation method for whole slide imaging. The effectiveness of the evaluation indices used in our experiments were confirmed through the result of linear regression analysis. Results of our experiments show that by incorporating the proposed image quality evaluation method, quality of whole slide images improves.

\section{Whole Slide Imaging telepathology for frozen section diagnosis: The University health network experience and lessons learned along the way}

\section{Andrew J. Evans \\ Laboratory Medicine Program, University Health Network, Toronto, ON, Canada}

Introduction: University Health Network (UHN) is a multi-site institution in downtown Toronto with a consolidated pathology department at Toronto General Hospital (TGH). Toronto Western Hospital (TWH) has no on-site pathologist and is located one mile from TGH. It generates 5-10 frozen sections (FS) per week, most coming from neurosurgery. Having pathologists travel to TWH to cover FS created challenges in terms of efficiency and consultation on difficult cases. Telepathology (TP) was identified as a solution and has been in use since 2004.

Methods: A team comprising a pathologist, a histotechnologist and an IT support person was formed in 2003 to select a TP system and validate it for FS diagnosis. After 18 months, the system went live using a robotic microscope (RM). In late 2006, parallel testing between the RM and a whole slide imaging (WSI) system (Aperio ScanScope CS) was carried out and a switch to WSI was made one month later.

Results: The RM was used to report 350 frozen sections. While the RM provided diagnostic accuracy equivalent to a light microscope, it took roughly 10 minutes to review FS slides. WSI was found to provide superior image quality, a user experience that more closely replicated light microscopy and a 4 to 5 -fold reduction in the amount of time required to review a slide. UHN pathologists have now reported over 2000 FS diagnoses by TP. WSI has provided accuracy equivalent to light microscopy with turnaround times between 14 and 16 minutes for single block FS and deferral rates of 5-8\%. Factors contributing to the success of the program include: a well-defined clinical application with an uncomplicated FS work flow, skilled histotechnologists and implementation period that allowed eam members to build confidence in the technology. Consistently high quality frozen section slides produced by skilled histotechnologists are critical in order to achieve diagnostic image quality. UHN's TP program has expanded to provide FS coverage to other centers in Ontario.

Conclusions: UHN has found WSI to be safe, accurate and reliable for making remote FS diagnoses. Successful implementation requires effective planning and a willingness to adjust traditional routines without compromising quality.

\section{Promoting knowledge translation through telehealth evaluation: A strategic choice for telepathology projects}

J.-P. Fortin ${ }^{1}$, M.-P. Gagnon ${ }^{2}$, L. Lamothe ${ }^{3}$ and B. Têtu

${ }^{1}$ Faculty of Medicine, Laval University, and CSSS de la Vieille, Capitale University affiliated Center, Québec, Canada

${ }^{2}$ Faculty of Nursing, Laval University and CHUQ research center, Quebec, Canada

${ }^{3}$ Faculty of Medicine, Montreal University, Population Health Research Institute, Quebec, Canada

${ }^{4}$ Faculty of Medicine, Laval University and CHUQ Research Center, Quebec, Canada

Background: Importance of Knowledge Translation (KT) to inform health decision regarding new technologies. The objective of the presentation is to provide an overview on how evaluation can be seen as a tool for promoting KT in the field of telehealth.

Methods: Based on the literature, field experiences of the investigators and a field study on the use of scientific evidence, we propose a framework that illustrates how evaluation can act as a KT tool at different stages of telehealth projects. We also present barriers and facilitators to the use of evaluation learning and process. Field experiences are related to investigators led "utilisation focussed evaluation" of 
various telehealth projects and to the development of innovative KT strategies. Both rely on close relationship between evaluators and decision makers.

Results: From the literature and field studies: there is a major lack of evidence uses in decision making for telehealth, and there is no unique solution for KT. Individual, organisational, contextual and evaluation factors are usually identified as facilitators or barriers. From the field experiences: A "Strategic framework for useful and used evaluation" was developed and is regularly used. It was more influential for implementation decisions making processes than for diffusion and sustainability of experimentations. KT initiatives were successful. There was a fast growing stakeholder's participation to an annual provincial forum for sharing and networking. Also a tool for change management was developed jointly with decision makers. It was adopted for national diffusion. There was also a political recognition of evaluation as a contributor for a success story of a major regional pre-hospital emergency care telehealth project.

Conclusions: This work illustrates several impacts that evaluation can have on the development, implementation, and diffusion of telehealth applications. Adaptation of the framework for an ongoing telepathology telehealth project in Quebec will be addressed.

\section{Applications of digitized whole-mount histopathology}

Martin J. Yaffe, Gina Clarke, Judit Zubovits, Chris Peressotti and Kela Liu

\section{Sunnybrook Research Institute and University of Toronto, Toronto, Canada}

In conventional histopathology, it is often necessary to attempt to correlate information from multiple adjacent sections to evaluate such features as tumour margins, multifocality, etc. We have developed techniques to allow entire sections of tissue specimens as large as $12.5 \mathrm{~cm} \times 17 \mathrm{~cm}$ to be produced on a single slide, digitized, viewed and analyzed.

For large, flaccid specimens, the resected tissue is placed in a gel which solidifies to support the tissue and approximately retain its in vivo conformation. Breadloafing is performed on a commercial slicer to facilitate fixation, which is performed with microwave assistance. Specialized techniques have been devised to avoid or correct for tissue shrinkage and to keep overall processing time within acceptable limits.

Digitization is carried out using the TissueScope (Huron Technologies, Inc., Waterloo, ON), which incorporates laser scanning and proprietary optics to capture brightfield or fluorescence images. For the largest slides, approximately 64 GB of image data are produced. A specialized viewing application, SEDEEN, was developed for convenient access to the image. It incorporates a hierarchical data structure for fast loading of images as well as a number of tools to manipulate the images, perform registration and quantitative analysis. By acquiring sequential sections at intervals appropriate for the specific application, three-dimensional analysis, e.g., of tumour burden, can be performed.

The technique was developed initially for evaluating the adequacy of surgical margins in breast lumpectomy specimens. However, it has since been found to be useful for prostate, and in research applications for vascular samples and animal hearts. An important application is in co-registering the whole-mount images with new targeted or functional in vivo imaging techniques to validate the latter with reduced sampling bias. One example is the evaluation of a laser-based imaging technique for monitoring response to neoadjuvant therapy in locally advanced breast cancer, where information reporting on tumour response from laser images acquired at various time points during the course of therapy is correlated the ground truth on whole-mount sections produced at mastectomy. We are currently engaged in studies to evaluate the accuracy of pathology assessment on whole-mounts versus conventional sized slides.

\section{Whole Slide Images and digital media in pathology education, testing, and practice- the Oklahoma experience}

Kar-Ming Fung ${ }^{1}$, Lewis A. Hassell ${ }^{1}$, Michael L. Talbert ${ }^{1}$, Allan F. Wiechmann ${ }^{2}$, Brad E. Chaser ${ }^{1}$ and Joel E. Ramey ${ }^{3}$

${ }^{1}$ Department of Pathology, University of Oklahoma Health Sciences Center, Oklahoma City, OK, USA

${ }^{2}$ Department of Cell Biology, University of Oklahoma Health Sciences Center, Oklahoma City, OK, USA

${ }^{3}$ College of Medicine, University of Oklahoma Health Sciences Center, Oklahoma City, OK, USA 
Background: The University of Oklahoma Health Sciences Center has seven health science colleges and two campuses (Oklahoma City and Tulsa). The main campus (Oklahoma City) is part of the 19-institution, 275-acre Oklahoma Health Center which includes adult, children's, and Department of Veterans Affairs hospital buildings, an eye institute, a cancer center, multiple clinics, facilities for training, simulation, education, and research. Modern communication technologies are critical for operations. In the past nine years, we have built an Internet and whole-slideimage-(WSI)-based platform for education and practice in pathology and histology.

Methods: Our educational platform uses a modular approach which includes quizzes, text pages, challenging cases, still images, WSI of normal histology, common and uncommon pathologic entities. Each module contains a small but complete set of educational materials such as a teaching case. These modules can be integrated into lecture notes, used for selfstudy, shown in lecture halls and used in small group discussions for a broad range of students and trainees with different needs and at multiple levels. Clinically, we actively use WSI in the archiving of consultation slides, for tumor boards, for quality control of immunohistochemistry, and for quantitative image analysis. We have also investigated the use of WSI in evaluation of the competency of residents and new faculty, and also in remote diagnosis of frozen sections.

Results: Our growing educational platform currently contains 298 histology WSIs, 119 pathology and neural histology WSIs, 478 quizzes, 102 challenging cases, didactic text pages, and hundreds of still images. The majority of these materials are opened to the public (http://www.oumedicine.com/body.cfm?id =2511). WSI greatly facilitates pathologic correlation clinically and academically, and is well accepted by clinical staff.

Conclusions: No single medium can fulfill all of the complex requirements for education and clinical practice. WSI is a powerful addition to our text- and stillimage-based platform. It reduces costs while it increases access - important issues for less wealthy regions. Global sharing of slides and their annotations becomes straightforward, particularly with future translations. Digital technologies greatly enhance interactions between students and instructors and between clinicians and pathologists. We anticipate an explosive impact of WSI in the near future.

\section{Digital pathology - implementation challenges in developing countries context}

Paul Fontelo ${ }^{1}$, John Faustorilla ${ }^{2}$, Alex Gavino ${ }^{1,2}$ and Alvin Marcelo ${ }^{1}$

${ }^{1}$ National Library of Medicine, Bethesda, MA, USA

${ }^{2}$ National Telehealth Center, University of the

Philippines College of Medicine, Manila, Philippines

Introduction: Learning pathology in a developing country context is challenging for several reasons. The expense and maintenance of optical microscopes is often more expensive. As a consequence, many students have to share microscopes in the teaching laboratory. The faculty-to-student ratio is also higher.

On the other hand, attempts at augmenting pathology teaching with computers have faced challenges. Cost of equipment is substantial and students' access to workstations is limited especially after class hours. Access to the Internet on academic networks is often slow and expensive. They are also subject to frequent power outages and adverse weather events.

Methods: Using iPad devices, 50 medical students (MSIII, 6\%, MSIV 94\%) evaluated their experience and access speed to virtual slides via Internet/cloud to a server at the US National Library of Medicine and via a local archive using a 5-point Likert scale (1-Strongly Disagree, 5-Strongly Agree).

Results and discussion: The results are summarized in the Table below. Although access to the Internet in the Philippines is widespread, the speed in most places is still less than $1 \mathrm{Mb} / \mathrm{s}$ in the majority of the country. The network in the medical school is rated at $8 \mathrm{Mb} / \mathrm{s}$ but desktop and wireless access is less than $1 \mathrm{Mb} / \mathrm{s}$.

\begin{tabular}{llll}
\hline Indicators & $\begin{array}{c}\text { NLM } \\
\text { server }\end{array}$ & $\begin{array}{l}\text { Local server } \\
\text { (Philippines) }\end{array}$ & $p$-value \\
\hline $\begin{array}{l}\text { The site is easy to } 4.04 \\
\text { access using an iPad }\end{array}$ & 4.62 & $<0.0001$ \\
$\begin{array}{l}\text { The site is easy to } \\
\text { navigate using an iPad }\end{array}$ & 3.82 & 4.5 & 0.0001 \\
$\begin{array}{l}\text { I see myself using } \\
\text { this site to address my }\end{array}$ & 3.98 & 4.42 & 0.0048 \\
$\begin{array}{l}\text { academic needs } \\
\begin{array}{l}\text { The site loads fast } \\
\text { enough to display a } \\
\text { clear image }\end{array}\end{array}$ & 3.86 & 4.52 & 0.0003 \\
\hline
\end{tabular}


Conclusion: Our results show that access to both servers was satisfactory but the local server was faster and preferred by the participants in this study. Virtual slides, accessible through a local network may enhance student learning in developing countries.

\section{Static digital telepathology: A model for a regional diagnostic and educational support network for pathologists in the developing world}

\section{Aliyah R. Sohani \\ Department of Pathology, Massachusetts General Hospital, Boston, MA, USA}

Background: The practice of pathology in the developing world presents challenges in terms of limited resources, shortages of subspecialists and trained laboratory personnel, and lack of continuing education programs. Telepathology holds promise as a means of diagnostic support, second opinions, and ongoing training. We report our experience with a static digital telepathology program established to support a regional network of 4 hospitals in Kenya and Tanzania, including 1 large tertiary care center with access to limited immunohistochemistry testing and 3 smaller hospitals staffed by solo pathologists.

Methods: Multiheaded teaching microscopes equipped with mounted digital cameras were donated to the 4 participating centers in East Africa. Local pathologists were given on-site training on image acquisition. Static images were uploaded to iPath open source telepathology servers. A US-based pathologist reviewed images in consultation with subspecialist colleagues, in order to provide both diagnostic and educational support to submitting pathologists as the program's main objective.

Results: Over a period of 3 years, 106 cases were submitted for second opinion consultation, including 27 dermatopathology cases $(25.5 \%) ; 13$ cases each $(12.3 \%)$ in hematopathology, cytopathology, and bone and soft tissue pathology; 8 cases each (7.5\%) in gastrointestinal, gynecologic, and head and neck pathology; and 7 breast pathology cases $(6.6 \%)$. The remaining 9 cases $(8.5 \%)$ comprised neuropathology, genitourinary, endocrine, pulmonary and medical renal pathology. Static images enabled a complete or partial diagnosis in 97/106 cases (91.5\%). Among partially diagnostic and non-diagnostic cases, factors precluding a definitive diagnosis included absence of confirmatory immunohistochemistry or flow cytometry, technical issues (air-drying artifact, tangential tissue sectioning, or poor image quality), or lack of sufficient clinical history. Responses posted to completely and partially diagnostic cases included a diagnosis, discussion of the differential diagnosis, and additional information (e.g., diagnostic pearls/pitfalls, references/publications about the entity).

Conclusion: Static digital telepathology is a simple and cost-effective means to provide both diagnostic and educational support to pathologists in the developing world, particularly in subspecialty areas that lack dedicated fellowship training programs in the region. Factors precluding a definitive diagnosis are mainly technical in nature and may be overcome by additional training or building local capacity for ancillary testing.

\section{Virtual microscopy beyond the pyramids, applications of WSI in Cairo University for E-education \& telepathology}

Essam Ayad

Digital Pathology Unit, Cairo University, Cairo, Egypt

Telepathology, the practice of pathology at a long distance, has advanced continuously since 1986 . Today, fourth-generation telepathology systems, socalled virtual slide telepathology systems [WSI], are being used for education applications. Both conventional and innovative surgical pathology diagnostic services are being designed and implemented as well. We have a successful experience in Egypt in applying the static \& dynamic techniques in a pilot project between the Italian Hospital in Cairo (NPO) and the Civico Hospital in Palermo. This project began in 2003 and continued till now. In 2004, centers in Venice, London and Pittsburgh participated actively in our project. During the past seven years we consulted on many problematic pathological cases with these different specialized pathological centers in Italy, UK \& USA. In addition to the highly specialized scientific value of consulting on the cases and exchanging knowledge, we saved a lot of time and money and succeeded in providing our patients with a better medical service. In view of this success we have already established a new Digital Telepathology unit 
(DTU) in the pathology department, Cairo University, using the latest technique of telepathology which is Whole Slide Imaging (WSI) since one year. This unit is considered the first Digital pathology unit in all the universities of the whole Middle East. During the passed two years we established a digital pathology library for the under graduate students using the WSI technique and changed the teaching method of the histopathology slides to be completely digital. We are building another digital pathology library [for post graduate candidates] which will be available to all pathology candidates in Egyptian universities \& universities in the surrounding Arabic countries. We are also creating a digital pathology network between pathology centers in the Middle East for exchanging knowledge \& telepathology. Moreover we began a new collaboration with UCDMC Department of Pathology, Sarcamento in the field of telepathology to exchange experiences \& teleconsultation.

\section{History, experiences, and perspectives of expert consultation in diagnostic surgical pathology}

Gian Kayser ${ }^{1}$, Stephan Borkenfeld ${ }^{2}$ and Klaus Kayser ${ }^{3}$

${ }^{1}$ Institute of Pathology, University of Freiburg, Freiburg, Germany

${ }^{2}$ IAT, Heidelberg, Germany

${ }^{3}$ Charite, University of Berlin, Berlin, Germany

Background: In addition to frozen section services, expert consultation (off line telepathology) was the first application of digital pathology in tissue - based diagnosis (diagnostic surgical pathology). Starting with closed fixed client - expert connections the first applications have been derived from "color - fax -machines" in the early 1990s. The implementation of the Internet and email configuration replaced the first systems by attachments to emails. At the beginning of this century three known open and flexible forum systems have been developed (iPATH, University of Basel, Switzerland, M. Oberholzer; UICC-TPCC (Telepathology Consultation Center of the Union International against Cancer, Charite, Berlin, Germany M. Dietel, P. Hufnagl), and the AFIP consultation system (Armed Forces Institute of Pathology, Washington, DC, USA, B. Williams). At present, all of them have been shut down due to various reasons. Only the iPATH system has been replaced by a commercially driven follower (Campus Medicus, Klughammer, Deggendorf, Germany).

Experiences: The first implementations were confronted with technical problems, velocity, stability of line connections, and features of microscope bound (analogue) cameras. Consecutively, experiments focused on velocity, size and quality of images transfer, often in relation to images of conventional microscopes.

Digitalization of the "telephone world" development of digital cameras and TV screens opened the door to routine expert consultation. Experiments analyzed image quality, diagnostic mistakes, and selection of field of view (region of interest, ROI).

Specific results: The diagnostic value of expert consultation varies with the specific task of the client (request of the pathologist). Most clients work in developing countries, and miss access to advanced diagnostic tools (specific stains, immunohistochemistry, molecular biology). Most of the involved experts are retired, and serve for consultation without any reimbursement. They have been organized in virtual pathology institutions (iPATH). Image quality, magnification and ROI selection still remain the main problem for certain clients. Most requests were answered by $2-3$ experts. Different expert opinions were seen in about $30 \%$ of the tasks. The average delay between task and answer accounted to 2-2 days. 


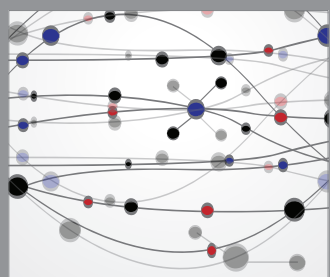

The Scientific World Journal
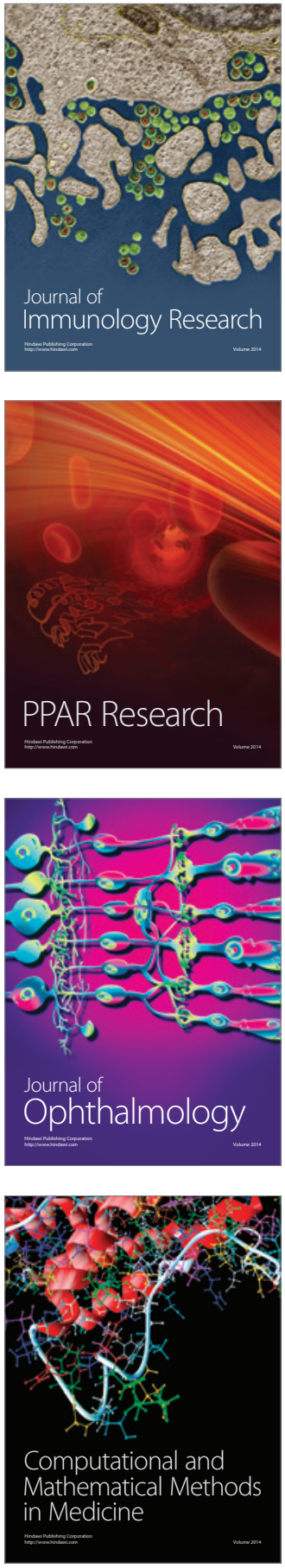

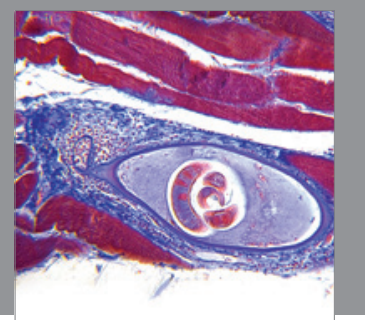

Gastroenterology

Research and Practice
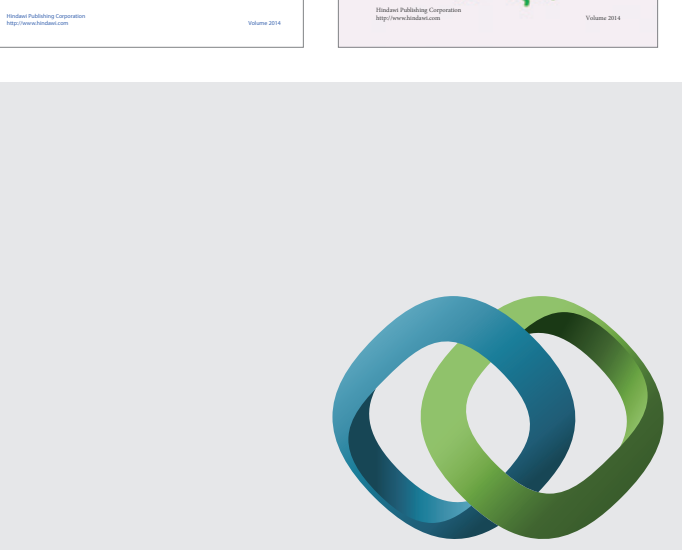

\section{Hindawi}

Submit your manuscripts at

http://www.hindawi.com
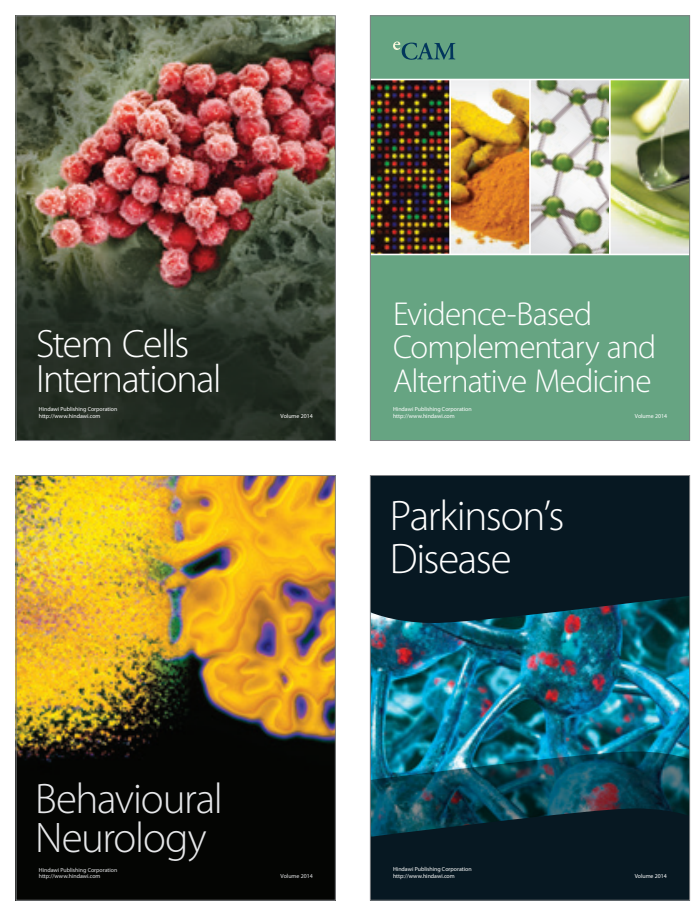

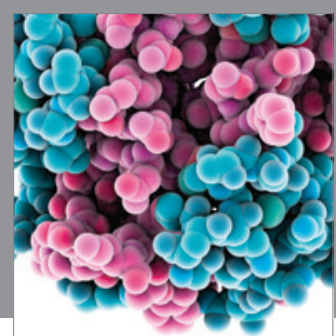

Journal of
Diabetes Research

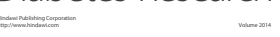

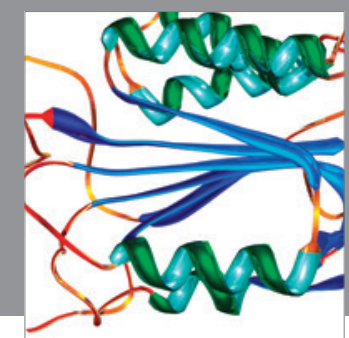

Disease Markers
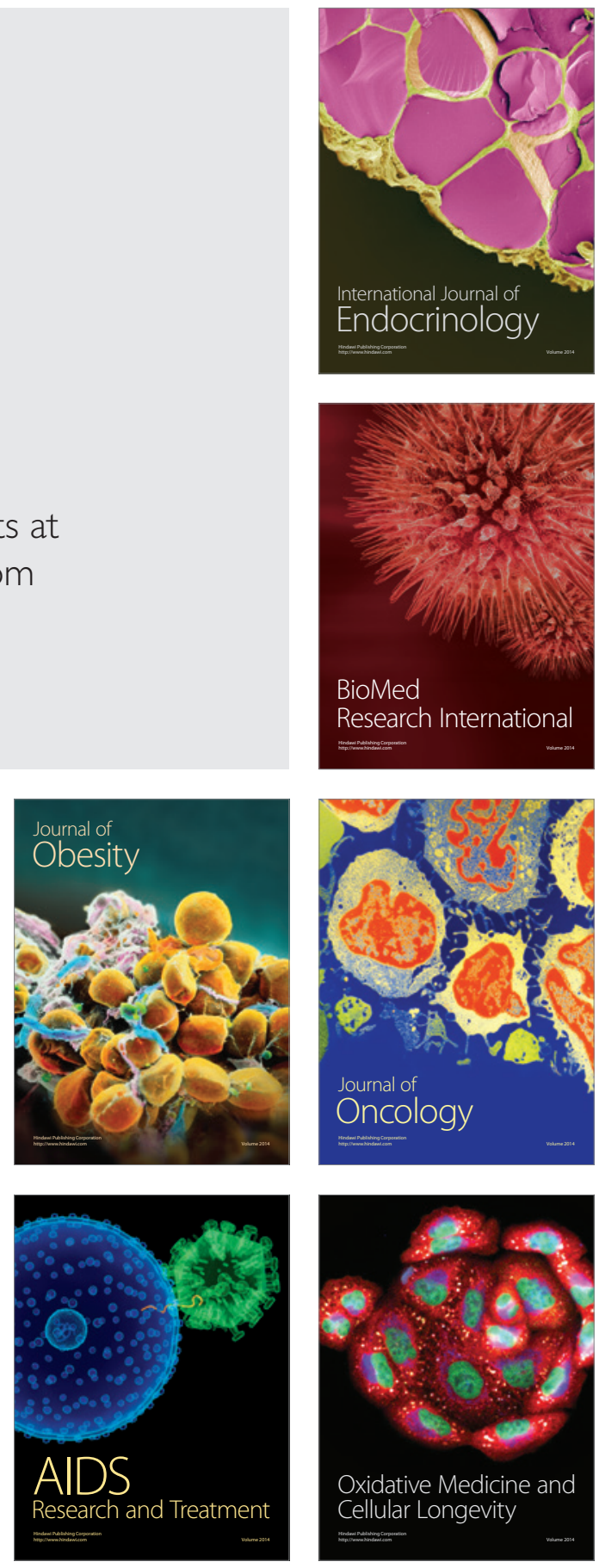